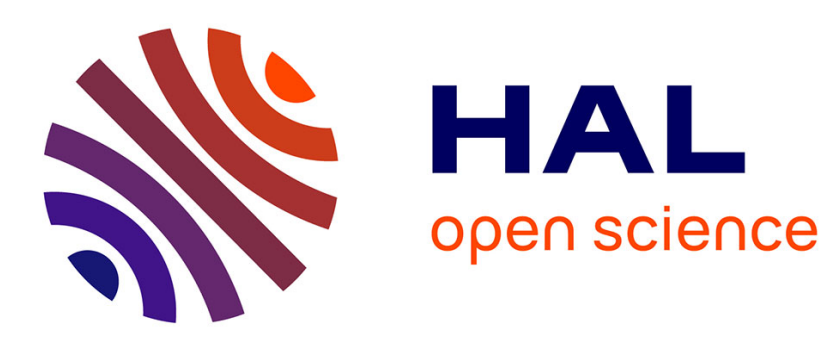

\title{
Biofluid Flow and Heat Transfer
}

Marc Thiriet, Wen-Hann Sheu, André Garon

\section{To cite this version:}

Marc Thiriet, Wen-Hann Sheu, André Garon. Biofluid Flow and Heat Transfer. Richard. W. Johnson. Handbook of Fluid Dynamics, CRC Press, 2016, 9781439849552. 10.1201/b19031-35 . hal-01393604

\section{HAL Id: hal-01393604 https://hal.sorbonne-universite.fr/hal-01393604}

Submitted on 9 Nov 2016

HAL is a multi-disciplinary open access archive for the deposit and dissemination of scientific research documents, whether they are published or not. The documents may come from teaching and research institutions in France or abroad, or from public or private research centers.
L'archive ouverte pluridisciplinaire HAL, est destinée au dépôt et à la diffusion de documents scientifiques de niveau recherche, publiés ou non, émanant des établissements d'enseignement et de recherche français ou étrangers, des laboratoires publics ou privés. 


\title{
Biofluid Flow and Heat Transfer
}

\author{
Marc Thiriet \\ University Pierre and Marie Curie \\ Wen-Hann Sheu \\ National Taiwan University \\ André Garon \\ Polytechnique de Montréal
}

September 1, 2012 


\section{Nomenclature}

A: cross-sectional area

A: aggregation rate

a: immersed particle acceleration

Be: Bejan number

Bo: Boltzmann constant

Br: Brinkman number

b: body force

C: stress tensor

C: heat capacity

C: charge density

$\mathrm{C}_{\mathrm{p}}$ : pressure coefficient

$c$ : concentration

c: wave speed

$\mathrm{c}_{\mathrm{p}(\mathrm{v})}$ : specific heat capacity at constant pressure (volume)

D: rate of deformation tensor

$\mathcal{D}$ : mass diffusivity

$D$ : drag coefficient

D: damage rate

$\mathcal{D}_{i j}$ : binary diffusion coefficient

$\mathcal{D}_{\mathrm{p}}$ : acoustic diffusivity

$\mathcal{D}_{\mathrm{T}}$ : thermal diffusivity

De: Dean number

Deb: Deborah number

d: local degradation rate

$d_{h}$ : hydraulic diameter

E: elastic component

$E$ : energy
Ec: Eckert number

I: metric tensor

$J$ : volume flux

$J_{e}$ : electric current density

$J_{\ell}$ : molar flux of species $\ell$

$L:$ vessel length

$M$ : metabolic rate

Ma: Mach number

$m$ : mass

$\mathrm{N}$ : aggregated erythrocyte number

Na: Navier number

$\mathrm{Nu}$ : Nusselt number

n: normal unit vector

$\mathcal{P}$ : membrane permeability

Pe: Péclet number

Pr: Prandtl number

$p$ : pressure

$Q$ : thermal energy

q: heat flux density

$q_{\mathrm{m}(\mathrm{v})}$ : mass (volume) flow rate

$q_{\text {met }}:$ metabolic heat source

$R$ : vessel radius

$R_{\mathrm{g}}$ : gas constant

Re: Reynolds number

$S$ : source term

S: acoustic streaming 
$F$ fragmentation rate

f: force vector

$f$ : frequency

$f\left(t, \mathbf{x}, v_{p}, r, \ldots\right)$ : probability density function

$\mathrm{G} i$ : tree generation $i$

$\mathcal{G}_{\mathrm{b}}$ : perfusion conductivity

$\mathcal{G}_{\mathrm{e}}$ : electrical conductivity

$\mathcal{G}_{\mathrm{h}}$ : hydraulic conductivity

$\mathcal{G}_{\mathrm{T}}$ : thermal conductivity

Gr: Graetz number

$g$ : gravitational acceleration vector

$H b$ : hemoglobin concentration

$H t$ : erythrocyte concentration

h: body's height

$h_{\mathrm{m}(\mathrm{T})}:$ mass (heat) transfer coefficient
Sc: Schmidt number

Sh: Sherwood number

St: Strouhal number

Sto: Stokes number

$T$ : temperature

$\mathrm{T}$ : period

$T^{\star}$ : time scale

t: tangential unit vector

$t$ : time

$\mathbf{u}$ : displacement

$V_{\mathrm{T}}$ : tidal volume

v: velocity vector

$W$ : external work

w: body's weight

$\mathbf{x}$ : space

\section{Greek symbols}
$\alpha$ : acoustic absorption coefficient
$\nu$ : kinematic viscosity
$\Gamma$ : boundary surface
$\mu$ : dynamic viscosity
$\gamma$ : modulation rate
П: osmotic pressure
$\dot{\gamma}$ : shear rate
$\rho$ : mass density
$\delta$ : boundary layer thickness
$\tau$ : time constant
$\kappa_{\mathrm{d}}$ : solute drag reflection coefficient
$\chi$ : mole fraction
$\kappa_{\mathrm{o}}$ : osmotic reflection coefficient
$\Omega$ : domain volume
$\omega$ : pulsation 


\section{Subscripts}

$\boldsymbol{\bullet}_{a}$ : arterial

-aw: airway

$\bullet_{b}$ : blood

$\bullet_{b r}$ : branch

$\bullet_{c}$ : curvature

- conv $_{\text {: convection }}$

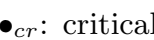

$\bullet_{d}$ : drag

- diff : diffusion (heat conduction)

$\bullet_{e}$ : external

$\bullet_{\text {eff }}$ : effective

- ent $_{\text {: entry }}$

- evap: evaporation

$\bullet_{i}$ : airway generation label

- $_{i d}$ : immersed droplet

$\bullet_{\ell}$ : chemical species label

$\bullet_{l w}$ : lumen-wall difference

$\bullet_{m}$ : mass
- met $_{\text {: metabolic }}$

$\bullet_{o}$ : osmotic

$\bullet_{p}$ : pressure

- $_{q}$ : cross-sectional average

- rad heat radiation

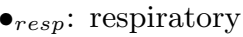

$\bullet_{S}$ : Stokes (unsteady) layer

$\bullet_{s}$ : source

- st: steady

$\bullet_{T}$ : thermal

$\bullet_{t}$ : trunk

$\bullet_{\text {tis }}$ : tissue

- $V M$ : von Mises (stress)

$\bullet_{v}$ : volume

$\boldsymbol{\bullet}_{w}$ : wall

$\bullet_{0}$ : low shear rate

$\bullet_{\infty}$ : high shear rate

\section{Superscripts and miscellaneous}

$\bullet \star$ : scale

$\overline{\mathbf{\bullet}}$ : time mean

๑: peak value

๑: dimensionless quantity

$<\bullet>$ : space average

\section{Mathematical notations}

$D_{t}$ : material derivative $(D / D t) \quad \partial_{\mathrm{t}}$ : time derivative $(\partial / \partial t) \quad \partial_{\mathrm{i}}$ : space derivative $\left(\partial / \partial x_{i}, i=1,2,3\right)$

$\nabla$ : gradient operator

$\nabla \cdot:$ divergence operator

$\nabla^{2}$ : Laplace operator 


\subsection{Introduction}

Major moving biological fluids, or biofluids, are blood and air that cooperate to bring oxygen to the body's cells and eliminate carbon dioxide produced by these cells. Blood is conveyed in a closed network composed of 2 circuits in series - the systemic and pulmonary circulation - - each constituted by arteries, capillaries, and veins, under the synchronized action of the left and right cardiac pumps, respectively. Air is successively inhaled from and exhaled to the atmosphere through the airway openings (nose and/or mouth). In the head, blood generates the cerebrospinal fluid in choroid plexi of all compartments of the ventricular system and receives it in arachnoid villi. Other biofluids are either secreted, such as bile from the liver and breast milk that both transport released substances with specific tasks, or excreted, such as urine from kidneys or sweat from skin glands that both convey useless materials and waste produced by the cell metabolism. In addition to the convective transport, peristalsis, which results from the radial contraction and relaxation of mural smooth muscles, propels the content of the lumen of the muscular bioconduit (e.g., digestive tract) in an anterograde direction.

Blood circulation and air flow in the respiratory tract are widely explored because of their vital functions. Note that inhaled air is transported through the respiratory tract by two processes: convection down to bronchioles and diffusion down to pulmonary alveoli. ${ }^{1}$ Furthermore, investigations of these physiological flows in deformable bioconduits give rise to models such as the Starling resistance that themselves become object of new study fields in physics and mechanics (e.g., collapsible tubes) as well as of new developments in mathematical modeling and scientific computing. Whereas fluid-structure interaction problems in aeronautics and civil engineering deal with materials of distinct properties, blood stream and vessel wall correspond to two domains of nearly equal physical properties, as both blood and vessels have densities close to that of water. New processing strategies must then be conceived.

\footnotetext{
${ }^{1}$ Pulmonary alveoli, sites of gas exchange between air and blood, coat the walls of the last generations of bronchioles in pulmonary acini and alveolar ducts. Diffusion of gas in air pipes and spaces relies on Maxwell-Stefan equations.
} 


\subsubsection{Stokes and Navier-Stokes equations}

The Navier-Stokes equations that govern Newtonian fluid flow are non-linear partial differential equations (PDE). They result from the balance of forces in isothermal flows of an incompressible fluid in pipes.

The main fluid variables, the velocity vector $\mathbf{v}$ and the stress tensor $\mathbf{C}$, use the Eulerian formulation. The main wall quantities are the stress $\mathbf{C}$ and displacement $\mathbf{u}$. At interfaces, fluid, compliant vessel walls, and possible flowing particle domains are coupled by continuity constraints. The set of conservation equations is closed by the relationships between the transmural pressure $p$ and the cross-sectional area $A$ (state law). ${ }^{2}$ The connected segments of the fluid circuit are decoupled with boundary conditions (BC) most often unknown. Constitutive laws depend on the microstructure of the materials.

Fluid flows are governed by mass, momentum, and energy balance principles, which are expressed by partial differential equations. The governing equations of an unsteady flow of an incompressible fluid (with mass density $\rho$, dynamic viscosity $\mu$, and kinematic viscosity $\nu=\mu / \rho)$ in an anatomical vessel conveyed with a velocity $\mathbf{v}(\mathbf{x}, t)(\mathbf{x}$ : Eulerian position, $t$ : time), are derived from the mass and momentum conservation. ${ }^{3}$

The equations of mass and momentum conservation of a moving incompressible fluid (i.e.,

\footnotetext{
${ }^{2} p=p_{\mathrm{i}}-p_{\mathrm{e}}\left(p_{\mathrm{i}}\right.$ : internal pressure), where the external pressure $p_{\mathrm{e}}$, the distribution of which is currently supposed to be spatially uniform, is assumed to be equal to zero. This assumption, which is a good approximation for superficial vessels, becomes questionable when the vessel is embedded in an environment that constraints the vessel. In addition, $p_{\mathrm{e}}$ can depend on time, such as in the thorax, where the pressure undergoes cyclic changes. Inflation and deflation of respiratory alveoli are, indeed, induced by nearly oscillatory variations in intrathoracic pressures generated by respiratory muscles, with a more or less strong gradient in the direction of the body height.

${ }^{3}$ Mass and momentum conservation equations are obtained from the analysis of evolution of involved quantities in an infinitesimal control volume, the so-called fluid particle (Part I, Chap. 3 General Equations of Newtonian Fluid Dynamics). The first basic postulate states that matter is neither destroyed nor created, meaning that any finite volume of matter neither disappears nor becomes infinite. The second basic postulate states that matter is impenetrable; any matter element does not share the same location with another element (continuity axiom). The following differential operators are used: (1) the gradient operator $\boldsymbol{\nabla}=\left(\partial_{1}, \partial_{2}, \partial_{3}\right)$, (2) the divergence operator $\boldsymbol{\nabla}$, and (3) the Laplace operator $\boldsymbol{\nabla}^{2}=\sum_{i=1}^{3} \partial_{i i}^{2}$. The gradient $\boldsymbol{\nabla} p$ of scalar $p$ is a vector of component $(\nabla p)_{i}=\partial_{i} p$. The gradient $\nabla \mathbf{v}$ of vector $\mathbf{v}$ is a second order tensor with component $(\nabla \mathbf{v})_{i j}=\partial_{i} v_{j}$.
} 
blood and air during rest breathing) are: ${ }^{4}$

$$
\begin{aligned}
\boldsymbol{\nabla} \cdot \mathbf{v} & =0 \\
\rho\left(\partial_{t}+\mathbf{v} \cdot \boldsymbol{\nabla}\right) \mathbf{v} & =\mathbf{b}+\boldsymbol{\nabla} \cdot \mathbf{C}
\end{aligned}
$$

where $\mathbf{C}=-p \mathbf{I}+2 \mu \mathbf{D}$ and the rate of deformation tensor $\mathbf{D}=\frac{1}{2}\left(\boldsymbol{\nabla} \mathbf{v}+(\boldsymbol{\nabla} \mathbf{v})^{T}\right)(\mathbf{I}$ : metric tensor) for a Newtonian fluid. In isothermal conditions, the equation set (1) leads to the simplified form of the Navier-Stokes equation in the absence of body forces $\mathbf{b}$ :

$$
\rho\left(\partial_{t} \mathbf{v}+(\mathbf{v} \cdot \nabla) \mathbf{v}\right)=-\nabla p_{\mathrm{i}}+\mu \nabla^{2} \mathbf{v}
$$

When the inertia term $(\mathbf{v} \cdot \boldsymbol{\nabla}) \mathbf{v}$ can be neglected, i.e., the diffusive term $\mu \boldsymbol{\nabla}^{2} \mathbf{v}$ is predominant, in the small blood vessels (arterioles) and bronchioles, the momentum conservation equation is called the Stokes equation:

$$
\partial_{t} \mathbf{v}+\nabla p_{\mathrm{i}} / \rho-\nu \nabla^{2} \mathbf{v}=0
$$

\subsubsection{Dimensionless form of Navier-Stokes equations}

The Navier-Stokes equations can be transformed into a dimensionless form using appropriate scales for the length $\left(L^{\star}\right.$; either the boundary layer thickness or the duct hydraulic radius for fully-developed flow), time $\left(T^{\star}\right.$; either the flow cycle period $\left[T^{\star} \equiv T\right]$ or the inverse of the flow pulsation $\left.\left[\left(T^{\star} \propto \omega^{-1}\right)\right]\right)$, velocity $\left(V^{\star}\right.$; either the mean or maximal cross-sectional velocity), and the pressure $\left(P^{\star}\right) .^{5}$

The classical formulation of the dimensionless Navier-Stokes equations are hence ob-

\footnotetext{
${ }^{4}$ The body force term (e.g., gravity and electromagnetic field during nuclear resonance imaging, especially using strong magnetic flux density) can be incorporated in the pressure term such that in the case of a gravity field:

$$
-\nabla p_{i}+\rho \mathbf{g}=-\nabla\left(p_{i}+\rho g z\right)=-\nabla \widehat{p_{i}} .
$$

${ }^{5}$ Therefore, $\tilde{t}=t / T^{\star}, \tilde{x}=x / L^{\star}, \tilde{v}=v / V^{\star}, \tilde{p}=p / P^{\star}$, and the dimensionless differential operators are then expressed by $\widetilde{\nabla}=L^{\star} \nabla$ and $\widetilde{\nabla^{2}}=L^{\star^{2}} \nabla^{2}$.
} 
tained: 6

$$
\underbrace{\frac{\rho V^{\star}}{T^{\star}}}_{T 1} \frac{\partial \tilde{\mathbf{v}}}{\partial \tilde{t}}+\underbrace{\frac{\rho V^{\star}}{L^{\star}}}_{T 2}(\tilde{\mathbf{v}} \cdot \widetilde{\nabla}) \tilde{\mathbf{v}}=-\underbrace{\frac{P^{\star}}{L^{\star}}}_{T 3} \widetilde{\nabla} \tilde{p}+\underbrace{\frac{\mu V^{\star}}{L^{\star}}}_{T 4} \widetilde{\nabla}^{2} \tilde{\mathbf{v}} .
$$

Note that for non-Newtonian fluid flow, the right hand side of equation 4 can be written as: ${ }^{7}$

$$
\widetilde{\nabla} \cdot\left(-\frac{P^{\star}}{L^{\star}} \tilde{p} \mathbf{I}+\frac{\mu_{\infty} V^{\star}}{L^{\star^{2}}} \tilde{\mu}\left(\widetilde{\nabla} \tilde{\mathbf{v}}+(\widetilde{\nabla} \tilde{\mathbf{v}})^{T}\right)\right)
$$

where $\mu_{\infty}$ and $\tilde{\mu}$ are the reference (at maximal shear rate) and dimensionless dynamical viscosity (Sect. 0.2.1). The expression 5 does not change the conclusion based on the classical formulation.

According to the predominant force type (convective inertial forces or viscous forces) the dimensionless coefficients related to the terms $T_{1}$ to $T_{4}$ of the equation 4 are displayed in Table 1. Both the Stokes and Strouhal number are important to assess the flow unsteadiness (Table 2). In steady flows, the pressure gradient balances the friction (and gravity when it has significant effect, as in blood vessels in standing position). In quasi-steady flows, the rate of change in boundary conditions are so slow that the momentum has time to diffuse during the flow period. In unsteady flows, the local inertia effects can be preponderant.

\subsubsection{Heat transfer equations}

The generation and transport of thermal energy in living tissue comprises several mechanisms, such as metabolism, conduction (or diffusion), convection, radiation, transport associated with mass transfer, and phase change (e.g., evaporation).

The transfer of thermal energy by diffusion between regions of a body's organ results from a temperature gradient to reach a thermal equilibrium. In fluids, heat conduction is due to diffusion and collisions of fluid molecules. The law of heat conduction (Fourier's law) states that the time rate of heat transfer through a material is proportional to the temperature

\footnotetext{
${ }^{6}$ The dimensionless Navier-Stokes equations suitable for a bend of uniform curvature in a single curvature plane can be found in $[1]$.

${ }^{7}$ A more general constitutive equation exists to model in particular Oldroyd fluids.
} 
Table 1: Dimensionless coefficients $\{\mathrm{T} i\}_{i=1}^{4}$ of the Navier-Stokes equation in the absence of body forces (equation 4). $\mathrm{C}_{\mathrm{p}}, \mathrm{Na}$, Re, Sto, and St are the pressure coefficient and Navier, Reynolds, Stokes, and Strouhal numbers $\left(T_{\text {diff }}^{\star}=L^{\star^{2}} / \nu ; T_{\text {conv }}^{\star}=1 / \omega ; \omega\right.$ : flow pulsation).

\begin{tabular}{lll}
\hline Coefficient & $\begin{array}{l}\text { Predominant convective inertia } \\
\left(\text { reference coefficient } \frac{\rho V^{\star^{2}}}{L^{\star}}\right)\end{array}$ & $\begin{array}{l}\text { Predominant friction } \\
\left.\text { (reference coefficient } \frac{\mu V^{\star}}{L^{\star^{2}}}\right)\end{array}$ \\
\hline $\mathrm{T} 1$ & $\frac{L^{\star}}{T^{\star} V^{\star}} \equiv \mathrm{St}$ & $\frac{T_{\mathrm{diff}}^{\star}}{T_{\text {conv }}^{\star}} \equiv \mathrm{Sto}^{2}$ \\
\hline $\mathrm{T} 2$ & $\frac{\rho L^{\star} V^{\star}}{\mu} \equiv \mathrm{Re}$ \\
\hline $\mathrm{T} 3$ & $\frac{P^{\star}}{\mu V^{\star} / L^{\star}} \equiv \mathrm{Na}$, \\
& $\frac{P^{\star}}{\rho V^{\star^{2}}} \equiv \mathrm{C}_{\mathrm{p}}$, & $\mathrm{Na} \equiv 1$, if $P^{\star}=\mu V^{\star} / L^{\star}$ \\
\hline $\mathrm{T} 4$ & $\mathrm{C}_{\mathrm{p}} \equiv 1$, if $P^{\star}=\rho V^{\star^{2}}$ & 1 \\
\hline & $\frac{\mu}{\rho L^{\star} V^{\star}} \equiv \mathrm{Re}^{-1}$ & \\
\hline
\end{tabular}

Table 2: Dimensionless governing parameters of arterial blood flow (cardiac frequency $1 \mathrm{~Hz}$, blood density of $1055 \mathrm{~kg} / \mathrm{m}^{3}$, dynamic viscosity $\left.3.5 \times 10^{-3} \mathrm{~Pa} \cdot \mathrm{s}\right)$.

\begin{tabular}{|c|c|c|c|c|}
\hline $\begin{array}{l}\text { Quantity } \\
\text { Parameter }\end{array}$ & \multicolumn{2}{|c|}{$\begin{array}{l}\text { Large } \\
\text { artery }\end{array}$} & $\begin{array}{l}\text { Small } \\
\text { artery }\end{array}$ & Arteriole \\
\hline Radius (mm) & \multicolumn{2}{|l|}{12.5} & 1.25 & 0.06 \\
\hline \multirow[b]{2}{*}{$\begin{array}{l}\text { Cross-sectional } \\
\text { velocity }(\mathrm{m} / \mathrm{s})\end{array}$} & Peak & Mean & Mean & Mean \\
\hline & 0.75 & 0.10 & 0.30 & 0.02 \\
\hline Reynolds number & 2700 & 370 & 110 & 0.4 \\
\hline Stokes number & 7 & 7 & 0.7 & 0.03 \\
\hline Strouhal number & 0.02 & 0.13 & 0.004 & 0.003 \\
\hline \multirow[t]{3}{*}{ Flow feature } & \multicolumn{2}{|c|}{ Pulsatile } & Quasi-steady & Steady \\
\hline & \multicolumn{3}{|c|}{ Homogeneous fluid } & Particle flow \\
\hline & \multicolumn{3}{|c|}{ Navier-Stokes equation } & Stokes equation \\
\hline
\end{tabular}


Table 3: Specific heat capacity at constant pressure of air and water at $27^{\circ} \mathrm{C}(300 \mathrm{~K})$ and $0.1 \mathrm{MPa}$; Source: [2]) and human blood (Sources: [3-6]; Ht: hematocrit).

\begin{tabular}{ll}
\hline Medium & $\mathrm{c}_{\mathrm{p}}$ \\
& $(\mathrm{J} /[\mathrm{kg} \cdot \mathrm{K}])$ \\
\hline Air (room conditions) & 1007 \\
Water & 4180.6 \\
\hline Blood & $3770[5]$ \\
& $3740(\mathrm{Ht}=30-32.4)[3]$ \\
& $3650(\mathrm{Ht}=41.6-42.4)[3]$ \\
Plasma & $3950[3]$ \\
Clotted blood & $3500 \pm 800[6]$ \\
\hline
\end{tabular}

gradient $(\nabla T)$ and the area normal to the transfer direction. The local heat flux density $(\mathbf{q})$ is then given by:

$$
\mathbf{q}=-\mathcal{G}_{\mathrm{T}} \nabla T
$$

where the thermal conductivity $\left(\mathcal{G}_{\mathrm{T}}\right)$ measures the tissue's ability to conduct heat.

The heat capacity $(\mathrm{C})$ of a biological tissue is the ratio between the amount of thermal energy $(Q)$ transferred to/from the tissue and the resulting increase/decrease in temperature $(\Delta T)$ of the biological tissue:

$$
\mathrm{C}=\frac{Q}{\Delta T}
$$

The heat capacity is defined at constant volume or constant pressure. Specific heat capacity, or thermal capacity, at constant volume or constant pressure is the amount of heat required to change the tissue temperature per unit mass to raise the tissue by 1 degree for $1 \mathrm{~kg}$ of matter:

$$
\mathrm{c}=\frac{\mathrm{C}}{m}=\frac{\mathrm{C}}{\rho V}
$$

( $\rho$ : material density). Values of the specific heat capacity at constant pressure of common fluids are given in Table 3.

The thermal conductivity $\left(\mathcal{G}_{\mathrm{T}}[\mathrm{W} /(\mathrm{m} \cdot \mathrm{K})]\right)$ measures the material's ability to conduct heat. Values of the thermal conductivity of common fluids are given in Table 4. 
Table 4: Thermal conductivity of air and water at $27^{\circ} \mathrm{C}(300 \mathrm{~K})$ and $100 \mathrm{kPa}$ (Source: [2]) and blood (Sources: [6, 7]; clot density 1.06-1.10 × 10 $0^{3} \mathrm{~kg} / \mathrm{m}^{3}$ ).

\begin{tabular}{ll}
\hline Material & $\begin{array}{l}\text { Thermal conductivity } \\
(\mathrm{W} /[\mathrm{m} \cdot \mathrm{K}])\end{array}$ \\
\hline Air & 0.02638 \\
Water & 0.61032 \\
Blood & $0.49-0.51$ \\
Plasma & $0.57-0.58$ \\
Clotted blood & $0.59 \pm 0.11$ \\
\hline
\end{tabular}

Table 5: Thermal diffusivity of selected media. The blood thermal diffusivity is computed for a density $\rho=1055 \mathrm{~kg} / \mathrm{m}^{3}$, a thermal conductivity $\mathcal{G}_{\mathrm{T}}=0.5 \mathrm{~W} /(\mathrm{m} \cdot \mathrm{K})$, and a specific heat capacity $c_{\mathrm{p}}=3700 \mathrm{~J} /(\mathrm{kg} \cdot \mathrm{K})$.

\begin{tabular}{ll}
\hline Material & $\begin{array}{l}\text { Thermal diffusivity } \\
\left(\mathrm{m}^{2} / \mathrm{s}\right)\end{array}$ \\
\hline Air & $2.256 \times 10^{-5}(1 \mathrm{MPa}, 300 \mathrm{~K})$ \\
Water & $1.465 \times 10^{-7}(1 \mathrm{MPa}, 300 \mathrm{~K})$ \\
\hline Blood & $1.3 \times 10^{-7}$ \\
\hline
\end{tabular}

The thermal diffusivity in a tissue $\left(\mathcal{D}_{\mathrm{T}}\right)$ is defined by:

$$
\mathcal{D}_{\mathrm{T}}=\frac{\mathcal{G}_{\mathrm{T}}}{\rho \mathrm{c}_{p}}
$$

Values of the thermal diffusivity of common fluids are given in Table 5.

In biofluids, the heat equation is modeled by a parabolic partial differential convectiondiffusion equation:

$$
\rho \mathrm{c}_{p} D_{t} T=\boldsymbol{\nabla} \cdot\left(\mathcal{G}_{\mathrm{T}} \boldsymbol{\nabla} T\right)+2 \mu \mathbf{D}: \mathbf{D}+S,
$$

where $D_{t}=\partial_{t}+(\mathbf{v} \cdot \boldsymbol{\nabla})$ is the material derivative and $S$ the source term (cellular metabolism). Most often, the second term (viscous dissipation of the flowing fluid) of the right hand side of equation 7 can be neglected compared to other equation terms. 


\subsubsection{Dimensionless form of heat transfer equations}

In the absence of heat source term other than viscous dissipation of the flowing fluid, the dimensionless form of the heat equation is:

$$
\underbrace{\rho c_{p} \frac{\Delta T}{T^{\star}}}_{T 1} \frac{\partial \tilde{T}}{\partial \tilde{t}}+\underbrace{\rho c_{p} \frac{V^{\star}}{L^{\star}} \Delta T}_{T 2}(\tilde{\mathbf{v}} \cdot \tilde{\nabla} \tilde{T})=\underbrace{\mathcal{G}_{\mathrm{T}} \frac{\Delta T}{L^{\star}}}_{T 3} \tilde{\nabla} \cdot(\tilde{\nabla} \tilde{T})+2 \underbrace{\mu\left(\frac{V^{\star}}{L^{\star}}\right)^{2}}_{T 4} \tilde{\mathbf{D}}: \tilde{\mathbf{D}},
$$

with the dimensionless temperature defined as $\tilde{T}=\left(T-T_{0}\right) / \Delta T\left(T_{0}\right.$ : body temperature $[310 \mathrm{~K}]) \Delta T$ the process temperature $\Delta T \sim M L^{\star} / \mathcal{G}_{\mathrm{T}}$ obtained via a dimensional analysis on fluxes $\left(M\right.$ : the metabolic rate $\left[\sim 50 \mathrm{~W} / \mathrm{m}^{2}\right.$ at rest $]$ ).

Table 6: Dimensionless coefficients $\{\mathrm{T} i\}_{i=1}^{4}$ of the heat equation in the absence of source terms (equation 8). Br, Ec, Pe, Pr, St, Sto are the Brinkman, Eckert, Péclet, Prandtl, Strouhal, and Stokes numbers.

\begin{tabular}{lll}
\hline Coefficient & $\begin{array}{l}\text { Predominant convection } \\
\left(\text { reference coefficient } \rho \mathrm{c}_{p} V^{\star} \Delta T / L^{\star}\right)\end{array}$ & $\begin{array}{l}\text { Predominant diffusion } \\
\left.\text { (reference coefficient } \mathcal{G}_{\mathrm{T}} \Delta T / L^{\star^{2}}\right)\end{array}$ \\
\hline $\mathrm{T} 1$ & $L^{\star} /\left(V^{\star} T^{\star}\right) \equiv \mathrm{St}$ & $L^{\star^{2}} /\left(\mathcal{D}_{\mathrm{T}} T^{\star}\right) \equiv \operatorname{Pr} . S t o^{2}$ \\
\hline $\mathrm{T} 2$ & 1 & $\left(L^{\star} V^{\star}\right) / \mathcal{D}_{\mathrm{T}} \equiv \mathrm{Pe}$ \\
\hline $\mathrm{T} 3$ & $\mathcal{D}_{\mathrm{T}} /\left(L^{\star} V^{\star}\right) \equiv \mathrm{Pe}^{-1}$ & 1 \\
\hline $\mathrm{T} 4$ & $\left(\nu / \mathrm{c}_{p}\right)\left(V^{\star} / L^{\star}\right) \Delta T^{-1} \equiv \operatorname{PrEc}$ & $\left(\mu / \mathcal{G}_{\mathrm{T}}\right)\left(V^{\star^{2}} / \Delta T\right) \equiv \mathrm{Br}$ \\
\hline
\end{tabular}

\subsubsection{Dimensionless governing parameters}

The formulation of the dimensionless equations depends on the choice of the variable scales

$\left(\bullet^{\star}\right)$. For example, the length scale is most often the vessel hydraulic radius $\left(L^{\star} \equiv R\right)$, the time scale is the flow period or, most often, the inverse of the flow pulsation, and the velocity 
scale either the mean $\left(V^{\star} \equiv \bar{V}_{q}\right)$ or the peak $\left(V^{\star} \equiv \widehat{V}_{q}\right)$ cross-sectional average velocity.

In Sect. 0.1.2, three dimensionless parameters were identified:

(1) the Reynolds number $\operatorname{Re}=V^{\star} L^{\star} / \nu$ is the ratio between convective inertia and viscous effects. When the flow is unsteady, the peak Reynolds number $\widehat{\operatorname{Re}}=\operatorname{Re}\left(\widehat{V}_{q}\right)$, characterizes the flow rather than the mean value $\overline{\operatorname{Re}}=\operatorname{Re}\left(\bar{V}_{q}\right)$. The study of flow stability relies on the peak Reynolds number computed with the unsteady boundary layer thickness, i.e., the ratio between the peak Reynolds number and the Stokes number $\left(\operatorname{Re}_{\delta_{\mathrm{S}}}=\operatorname{Re} / \mathrm{Sto}\right)$;

(2) the Stokes number Sto $=L^{\star}(\omega / \nu)^{1 / 2}$ is the square root of the ratio between time inertia $\left(\propto \rho \omega U^{\star}\right)$ and viscous effects $\left(\propto \mu U^{\star} / L^{\star^{2}}\right)$ as well as a length ratio Sto $=R / \delta_{\mathrm{S}}\left(\delta_{\mathrm{S}}\right.$ : Stokes boundary layer thickness; $\left.\delta_{\mathrm{S}}=(\nu / \omega)^{1 / 2}\right)$ and a time ratio $\operatorname{Sto}^{2}=\left(R^{2} / \nu\right) / T=T_{\text {diff }}^{\star} / T{ }^{8}$

(3) the Strouhal number St $=L^{\star} /\left(T^{\star} V^{\star}\right)$ is the ratio between time and convective $\left(\propto \rho U^{\star^{2}} / L^{\star}\right)$ inertia $\left(\mathrm{St}=\mathrm{Sto}^{2} / \mathrm{Re}\right)$.

Additional dimensionless parameters can be deduced from similar analyses.

(1) The Dean number De $=\left(R / R_{c}\right)^{1 / 2} \operatorname{Re}\left(R_{c}\right.$ : curvature radius of tube axis), for laminar flow in curved vessels, is the product of the square root of the vessel curvature ratio by the Reynolds number. ${ }^{9}$

(2) The modulation rate, or amplitude ratio, is used when the time-dependent component of the fluid flow is a sinusoid of amplitude $V_{\sim}$ superimposed on a steady one $\left(\gamma_{v}=V_{\sim} / \bar{V}\right)$ to assess the magnitude of the near-wall back flow during the bidirectional phase of the pulsatile flow. The lower the modulation rate, the smaller the retrograde flow.

(3) The Deborah number Deb $=\left(\tau V^{\star} / L^{\star}\right)=\tau / T_{\text {conv }}^{\star}$, an inverse Strouhal-like number, is the ratio between the time constant $(\tau)$ for aggregation of erythrocytes (or red blood cells)

\footnotetext{
${ }^{8}$ Both Stokes and Rayleigh boundary layers thickness are $\propto(\nu T)$. The Rayleigh boundary layer deals with a flow over a flat plate which suddenly moves in its own plane, with a constant speed (transient regime). The Stokes boundary layer deals with a harmonic motion of a flat plate in its own plane, with an angular frequency $\omega$ (periodic flow). The latter case is more relevant to physiological flows. Note that the Stokes number is also commonly called Womersley number and sometimes the Witzig-Womersley number in the literature.

${ }^{9}$ The Dean number is usually calculated in simple bends of constant curvature, such as those used in experiments or as simulation benchmarks. In image-based flow models, the vessel axis varies continually in every direction; consequently, the Dean number is not computed.
} 
and the flow time scale. When Deb $>1$, erythrocytes have enough time to aggregate.

(4) The Mach number is the ratio between the cross-sectional average velocity and the speed of propagation of pressure wave (Ma $=V_{q} / \mathrm{c}$ ), the wave speed depending on the vessel distensibility $\left(c^{2}=1 /\left[\rho\left(\partial\left[A / A_{0}\right] / \partial p\right)\right]\right)$.

(5) The Prandtl number is the ratio of the momentum diffusivity to thermal diffusivity $\left(\operatorname{Pr}=\nu / \mathcal{D}_{\mathrm{T}}\right)$ and the Péclet number for thermal diffusion in fluid flows is given by: $\operatorname{Pe}_{\mathrm{T}}=\operatorname{Re} \times \operatorname{Pr}^{10}$

(6) The Nusselt number is the ratio between convective and conductive heat transfer, i.e., the ratio between the product of the convective heat transfer coefficient and the flow characteristic length and the thermal conductivity of the fluid $\left(N u=h_{\mathrm{T}} L / \mathcal{G}_{\mathrm{T}}\right){ }^{11}$

(7) The Brinkman number $\left(\mathrm{Br}=\left(\mu V^{2}\right) /\left(\mathcal{G}_{\mathrm{T}} \Delta T\right)\right)$ is related to heat conduction from a wall to a flowing viscous fluid. The Brinkman number is $3.15 \times 10^{-3}, 5.04 \times 10^{-3}$, and $4.67 \times 10^{-3}$ for a large and small artery and arteriole, respectively. Hence the viscous dissipation term can be neglected.

(8) The Graetz number $\left(\mathrm{Gr}=\left(d_{h} / L\right) \mathrm{Pe}\right)$ determines the thermally developing flow entrance length in ducts. It can be defined as the ratio of the product of the mass flow rate per unit volume of a fluid times its specific heat at constant pressure to the product of its thermal conductivity and a pipe length.

(9) In fluid flows with heat transfer, the Bejan number is the dimensionless pressure drop along a channel of length $\mathrm{L}: \mathrm{Be}=\left(\Delta p L^{2}\right) /\left(\mu \mathcal{D}_{\mathrm{T}}\right)$.

(10) The Eckert number expresses the relation between the flow kinetic energy and enthalpy: $\mathrm{Ec}=V_{\infty}^{2} /\left(c_{p} \Delta T\right)$. It is used in momentum and heat transfer to characterize dissipation, especially in the case of immersed bodies in a compressible fluid flow $\left(V_{\infty}\right.$ : characteristic fluid velocity far from the body; $\Delta T$ : difference between temperatures of the fluid and body).

\footnotetext{
${ }^{10}$ Similarly, the Schmidt number is the ratio of momentum diffusivity to mass diffusivity $(S c=\nu / \mathcal{D})$ and the Péclet number for mass diffusion in fluid flows is given by: $\mathrm{Pe}_{\mathrm{m}}=\mathrm{Re} \times \mathrm{Sc}$.

${ }^{11}$ Similarly, the Sherwood number is the ratio of the convective to diffusive mass transfer $\left(S h=h_{\mathrm{m}} L / \mathcal{D} ; h_{\mathrm{m}}:\right.$ mass transfer coefficient).
} 


\subsubsection{Physiological boundary conditions}

Boundary conditions, as well as initial conditions, are required to solve the set of partial differential equations. Numerical simulations of the flow in a segment of physiological bioconduit network require the specification of boundary data on artificial boundaries of the computational domain, which limit the explored vascular district. The boundary of the fluid domain $(\Omega)$ is usually partitioned into three main types of surfaces: vessel entry $\left(\Gamma_{1}\right)$ and exit $\left(\Gamma_{2}\right)$ cross-sections and wall $\left(\Gamma_{3}\right)$. The classical no-slip condition is applied to the rigid vessel wall. On the other hand, in the case of a deformable wall, the moving boundary $\Gamma_{3}$ is the fluid-structure interface. Inlet and outlet boundary conditions influence the fluid dynamics and must then be set away from the region of interest. Moreover, upstream and downstream effects caused by any geometrical singularities such as bends must be incorporated; hence the $3 \mathrm{D}$ computational domain must contain proper boundary regions at some distance from the explored region to avoid numerical bias.

In the cardiovascular system, flow and pressure waves emanate from the heart and travel through the major arteries where they are damped, dispersed, and reflected due to changes in vessel caliber and wall properties as well as branchings. As a consequence, solutions to the governing equations of blood flow in large vessels depend on inlet and outlet impedances.

A time-dependent injection velocity $\mathbf{v}_{\Gamma}(t)$ can be prescribed, at least, at the inlet. Most often the spatial resolution of in vivo velocity measurements is indeed not high enough to provide velocity distribution at vessel ends. Velocity conditions can be obtained from Fourier transforms of in vivo ultra-sound or magnetic resonance (MR) flow signals. These measurements are commonly carried out more or less far from the computational domain entry and not necessarily in the same subject.

In the absence of measurements, the inlet velocity profile is given by the Womersley solution [8]. The Womersley solution corresponds to a Poiseuille flow superimposed to a harmonic flow, hence to a pulsatile fully-developed flow in a long, smooth, straight pipe of circular cross-section, a condition never encountered in the human body. On the other hand, time-dependent, uniform injection velocity is associated with an artificial high wall shear, 
hence vorticity, at the entrance station that is susceptible to induce larger flow separation if an adverse pressure gradient is set up.

Bulk interactions between the region of interest and network parts upstream and dowstream from it are currently neglected. In fact, at the outlet cross-sections, the flow distribution and pressure field remain unknown. The most common outflow boundary conditions for three-dimensional flow simulations are prescribed traction and velocity profiles: ${ }^{12}$

$$
(\mathbf{C} \cdot \mathbf{n}) \cdot \mathbf{n}=f_{0} ; \quad \mathbf{v} \cdot \mathbf{t}=0
$$

where $\mathbf{n}$ and $\mathbf{t}$ are the local unit normal and tangent vectors and the outlet pressure is approximately equal to $-f_{0}$. In the case of multiple duct exits, $f_{0}$ influences the flow distribution among the model branches. These standard boundary conditions at intlet(s) and outlet(s) of a small explored region of the vasculature do not take into account input and output impedances and then cannot predict accurately the flow behavior.

Artery walls can bear large displacements, as the arterial lumen can vary up to about $10 \%$ between the diastolic and systolic configuration. A multiphysics approach is then necessary to provide suitable boundary conditions both at the moving fluid-solid interface and domain inlets and outlets.

When physiological measurements cannot produce suitable boundary data, a mathematical description of the action of the reminder of the biofluid circuit on the studied region can be carried out [9-13]. A multilevel modeling couples 3D flow models (detailled models relying on PDEs) to OD (lumped-parameter models, or electrical analogs, described by ordinary differential equations). In the case of deformable bioconduits, 1D models are incorporated for adequate wave propagation.

\footnotetext{
${ }^{12}$ The outlet sections must be perpendicular to the local vessel axis and be short straight pipe exits to avoid pressure crossgradient.
} 


\subsubsection{Main solving procedures}

Because of the bioconduit architecture and flow conditions, the Navier-Stokes equations do not have analytical solutions. Numerical simulations that use a set of algebraic equations, which approximate the Navier-Stokes equations, are thus carried out. Computational fluid dynamics is indeed aimed at solving problems of the dynamics of single- or multiphase, compressible or not, Newtonian or not, steady or not, fluid flow alone or coupled to the solid wall mechanics as well as heat and mass transfer and associated phenomena such as chemical reactions.

Numerical modeling of physiological flows is often carried out to provide a complete description of the flow in a specific region. The local fields of hemodynamic variables are obtained using 3D simulations, based on the numerical approximation of the incompressible Navier-Stokes equations, in general, in a rigid domain computed from image processing.

Whatever the approximation method selected, once the physical and chemical processes are modeled and fluid properties determined, flow simulations require to mesh the geometry of interest, i.e., to split it into discrete, small, non-overlapping grid cells (also called numerical control volumes and geometrical elements). ${ }^{13}$ Numerical simulation accuracy should be independent of the mesh size. Simulations indeed should proceed through a rigorous verification and validation process using error estimation technique and error-based optimal grid adaptation technique. ${ }^{14}$ In addition, mesh adaptivity ensures suitable solutions of problems associated with propagation and wall motion.

Proper boundary conditions are specified at the boundaries of the computational domain and mapped to reference numbers assigned to boundary regions of the domain and possibly its subdomains.

Numerical methods approximate the fields of unknowns (e.g., velocity and pressure) by means of simple functions. Governing equations are discretized to get a set of algebraic

\footnotetext{
${ }^{13}$ Meshfree methods rely on a particle approach over the entire domain for calculations.

${ }^{14}$ Specifically, verification aims at proving that the equations of the mathematical model are solved correctly, whereas validation aims at comparing the solution obtained using the selected model with measurements to check the validity of numerical tests. Verification and validation are now standardized [14, 15]. Furthermore, many scientific journals such as the $A S M E$ Journal of Fluids Engineering "will not accept for publication any paper reporting the numerical solution ... that fails to address the task of systematic truncation error testing and accuracy estimation."
} 
equations. Simulations are then initiated and the set of algebraic equations that results from the approximation procedure of partial differential equations (PDE) is solved iteratively. ${ }^{15}$

Among many approximation and discretization procedures, ${ }^{16}$ two major discretization methods are used. The finite volume method (FVM) is based on integral conservation laws and ensures the flux continuity in each mesh cell $[16,17] .{ }^{17}$ The finite element method (FEM) relies on the discretization of the weak form of variational formulations of PDEs defined in finite dimensional spaces and ensuring a conservative solution, using a suitable choice of bases [18-21]. ${ }^{18}$ Problem unknowns are piecewise approximated (at the element level).

\subsubsection{Image-based computational model}

Computerized medical imaging provides subject-dependent 3D geometry of any body organ, in particular large bioconduits. Nowadays, numerical simulations are carried out after 3D reconstruction of bioconduits of interest to yield subject-specific computational domains. Input data for the surface reconstruction of the target organ usually come either from Xray computed tomography [22-24], magnetic resonance images [25-28], or 3D ultrasound images [29].

The common two-step technique to create a mesh from imaging data consists first in segmenting the selected organ in the images, and then using the segmentation surface to create the facetization. A set of metrics for variable shapes including the so-called elastic metrics that enable the identification of surfaces is given in [30].

\footnotetext{
${ }^{15}$ The numerical solution must converge toward the exact solution that corresponds to cells of infinitesimal size. Consistent schemes produce sets of algebraic equations equivalent to the original governing equations, when the mesh size tends to zero. Stability is associated with error damping as iterations proceed.

${ }^{16}$ The finite difference method (FDM) based on point samples at mesh nodes is the simplest technique to program. Truncated Taylor series expansion generate finite difference approximation. The spectral method expresses the problem unknowns in truncated Fourier series or series of Chebyshev polynomials and substitute the series into the set of PDEs to get a system of ordinary differential equations (ODE) that are solved. The boundary element method (BEM) uses an integral formulation to solve linear partial differential equations for which Green's functions can be calculated.

${ }^{17}$ Values of unknowns of flow phenomena (convection, diffusion, and source and sink terms) are computed at discrete nodes of control volumes of the mesh. This iterative solving technique deals with surface integrals on these discrete control volumes. Fluxes at the surfaces of each finite volume are conservative, the flux entering a given volume being that leaving the adjacent volume (conservativeness). The solution is bounded by the minimum and maximum values of the flow variable (boundedness). Transportiveness account for the directionality of influence in terms of relative magnitude of convection and diffusion.

${ }^{18} \mathrm{~A}$ linear combination of basis functions is piecewise continuous (linear, quadratic, or polynomial) or not over each finite element. A residual is computed to determine the errors.
} 
The automatic mesh generator must be able to cope with such surface, which is frequently full of gaps, overlaps, and other imperfections. Various algorithms have been proposed to reconstruct a polygonal model (a piecewise linear approximation) depending on the nature of the sampled data (series of slices, range images, point clouds, etc.). Slice connection algorithms work for a series of planar parallel sections of the target vessel. At first, a closed contour is extracted in each slice, then contours are connected to each other between each pair of adjacent slices to reconstruct the surface of the bioconduits [31, 32].

Marching-cube approach attempts to extract an implicit surface from a 3D range image based on a "voxelhood" analysis. Delaunay tetrahedralization algorithms first generate 3D triangulation over a point cloud and then extract a bounded surface triangulation from this set of tetrahedra using suitable topological and geometrical criteria [33].

Level set methods can detect an interface between a vessel and the surrounding tissues. On the other hand, deformable models employ an average template mesh for explored bioconduits and performs an elastic deformation of the template onto the image set until the vessel surface is matched [34]. In addition, the vascular segment of interest can be placed in a cube that is subdivided into octants of length scale determined by the mesh size to suit the possible caliber changes [35]. The octants containing the vessel walls are trimmed to match the wall surface with a given tolerance level. Smoothing can then be performed. The octants within the vessel are subdivided into tetrahedra.

Smooth implicit functions for 3D reconstruction have been associated with spectral/hp high order elements for blood flow computations [36]. Bicubic spline patches, which are interactively defined and projected onto the implicit surface, serve as the initial element for meshing [37]. Mesh size and shape optimization is determined by the eigenvalues of the Hessian matrix of an implicit function.

Snakes, or active closed contours (curves) defined within an image domain, can be used for image segmentation [38]. Snakes move under the influence of internal forces coming from the curve and external forces computed from the image data that are defined so that the snake conforms to the object boundary [39]. 
Last, but not least, bioconduit modeling can be based on determination of its axis. Once the set of axes is known, a "response function" is computed for each vessel slice from a vector rotating around the axis point and the intensity gradient. The voxel positions for which the response function is maximum give the vessel contour in the investigated slices when the axis is correctly determined [40].

\subsubsection{Biofluid flow features}

Biofluid flows share common features. Due to the architecture of the bioconduit network, the flow is three-dimensional and developing, or unestablished. Therefore, the cross-sectional velocity distribution is associated with a growing boundary layer and an axial, non-linear pressure drop. The flow generated by anatomical pumps (heart and thoracic cage) is quasiperiodic.

These flow features as well as the architecture of the vessel network and bioconduit wall deformability influence the flow regime (laminar, transitional, or partially turbulent). Moreover, the wall compliance can explain in some circumstances the occurrence of trans- and supercritical flows, when the flow velocity equals and becomes greater than the speed of propagation of pressure waves mainly associated with the wall distensibility [41].

\section{Three-dimensional flow}

Convective transport in physiological systems, primarily the well explored blood circulatory and respiratory systems, occurs in bioconduit network that are characterized by various types of geometrical singularities:

(1) curvatures in all directions that change continuously;

(2) huge number of lateral branches, junctions, ${ }^{19}$ and terminal bi- and trifurcations; and

(3) convergent and divergent segments, such as taper of a long vessel after successive

\footnotetext{
${ }^{19}$ The venous network consists of junctions of draining venules and small, mid-size, and large veins. The arterial bed possesses at least one junction, the basilar trunk that arises from the merging of vertebral arteries.
} 
branchings and prints on vessels imposed by apposed organs in normal conditions as well as in- and extrinsic pathological stenoses.

Out-of-plane curved vessel axis causes helical fluid movement. Flow variables thus evolve in three-dimensional fields confined by bioconduit walls of given rheology.

\section{Developing flow}

Any flow entering in a pipe experiences a resistance from the wall. The strongest effect of viscosity is exerted in the fluid boundary layer in the near-wall region of the vessel lumen. In the entry, or entrance, length $\left(\mathrm{L}_{e}\right)$ of the vessel, the momentum diffuses toward the vessel axis and the boundary layer grows. The boundary layer thickness is the distance from the wall to a point where the flow velocity becomes equal to the core-flow velocity, in which the fluid behaves as an inviscid material. A fully-developed flow that appears when the boundary layer reaches the vessel axis is characterized by an axially invariant velocity crossdistribution and a linear axial pressure drop (constant pressure gradient) [42]. In any case, in a deformable bend the short distance between two branch points prevents the appearance of a fully-developed flow.

\section{Unsteadiness}

Blood circulation is characterized by a bulk unidirectional transport during most of the cardiac cycle (in arteries, blood flows away from the heart). However, bidirectional flow (fluid flows in the streamwise direction in the core lumen and in the opposite direction in the near-wall region) appears in elastic arteries (close to the heart) during the diastole, except in carotid arteries of the cerebral circulation. Moreover, the blood pulsatile flow reverses its direction throughout the entire cross section toward the heart in some proximal arteries such as the aorta.

In the respiratory tract, air flows alternatively through the same air bioconduits from nose (or mouth) to pulmonary alveoli during inhalation and exhalation. This oscillatory-like 
motion in the open respiratory tract (in opposition to the closed blood circuit) transports air from the atmosphere to alveoli and conversely.

Whereas pumps are incorporated in the blood circulation, air travels back and forth in the respiratory tract, closed ends of which are located in the musculo-osseous thoracic cage that serves as ventilatory pump. Motions of the thoracic wall successively pressurize and depressurize the cavity content, hence inflating and deflating intrathoracic air spaces. The resulting changes in intra-alveolar pressure drive air motions between atmosphere and deformable alveoli.

\section{Deformable wall}

Biofluids are transported in deformable bioconduits. Airways downstream from bony structure, i.e., the pharynx, larynx, trachea, and large bronchi may deform in deep breathing. Furthermore, during forced expiration, the large intrathoracic airways undergo a strong collapse.

During the contraction of the ventricular myocardium, the ejected systolic blood bolus is partly transmitted in the perfusion network, but also stored in large elastic arteries near the heart exit. During the following diastole (ventricular myocardium relaxation), the stored blood is restituted and converts the starting-stopping flow downstream from the ventriculoarterial valves into a pulsatile stream in downstream arteries. The locally and transiently stored blood flow during the systole is transferred in both up- and downstream directions during the following diastole. Upstream blood flux permits, in particular, coronary irrigation, hence heart perfusion. Whereas any artery transiently dilates during the passage of the travelling pressure wave, veins of inferior limbs experience a partial collapse during contraction of skeletal muscles between which they run, especially during walking. 


\section{Living, sensing, and reacting wall}

Large bioconduits, such as arteries, veins, trachea, and bronchi, possess a multi-layered wall. Walls of arteries and veins are composed of an inner intima, a media, and an outer adventitia. Each wall layer is made of a composite material. The adventitia contains a perfusing microvasculature (vasa vasorum) and is innervated. The media determines the luminal caliber via the contraction or relaxation of its smooth muscle content. The intima is made of a single, wetted layer of endothelial cells over a thin sheet of connective tissue. Whereas smooth muscle cells sense azimuthal and longitudinal tension induced by the blood pressure (stress component normal to the vessel wall), endothelial cells sense the local shear (tangential stress component) applied by the flowing blood. These cells thus contribute to the regulation of the contraction-relaxation state of the smooth muscle cells of the media, hence the caliber of the vessel lumen. The flow can then adapt to maximize the organ perfusion and minimize the heart afterload.

\subsubsection{Flow regime}

In the laminar regime, steady or not, the fluid moves in a somewhat orderly fashion. However, when the Reynolds number increases, some regions of the flow become chaotic. The turbulence is intermittent and alternates with quiescent laminar regions. This transitional regime may evolve to a fully turbulent state, a random motion being observed everywhere within the flow.

The fluid flow regime depends on the Reynolds number (Re). If the value of Re is smaller than a critical value $\left(\mathrm{Re}_{\mathrm{cr}}\right)$ that should be appropriate to flow conditions (time-dependent flow) and bioconduit geometry (curvature), then the flow is laminar. On the other hand, when $R e>R_{e r}$, then the flow becomes turbulent. In straight pipes, $\operatorname{Re}_{\text {cr }}$ can be equal to $10^{5}$ (not the usual value 2300 found in any textbook) in carefully performed experiments. The values for physiological flows remain unknown.

In a stable flow, any small disturbance is damped. Strong local pressure gradients, velocity 
profile inflections, and shear reversals affect the flow stability. Flow instabilities can be related to vortex production; vortices arise from high-vorticity regions. Sheared vortex can then be stretched and rotate. Self-modulated perturbations can lead to evolving smallscale structures. In an intermittent (disturbed) flow, transient instabilities decay as they

propagate downstream, due to the dissipative action of viscous forces. Amplification of vorticity perturbations in flow can lead to a transitional flow.

Periodic flows are transiently stable if a disturbance grows during a part of the cycle and decays. On the other hand, periodic flows are unstable when the disturbance grows during each period. Periodic flow patterns can be classified into four main types:

(1) laminar;

(2) disturbed with small amplitude perturbations;

(3) intermittently turbulent flow in which high-frequency velocity fluctuations occur at the beginning of the deceleration phase, increase, and dissipate prior to or during the subsequent acceleration phase; and

(4) fully developed turbulent flow, high-frequency velocity fluctuations existing during the whole flow cycle.

\subsubsection{Fluid-structure interaction}

Simulations of biological flows often rely on multiphysics modeling and solver-coupling platforms, in particular on fluid-structure interaction in deformable conduits, especially in large blood vessels (particularly in elastic arteries near heart exits) as well as bronchi during large-amplitude breathing at more or less high frequency, in addition to flow of deformable red blood capsules (cells) in the microvasculature and transport of sprays in the respiratory tract. Moreover, simulation of venous and cardiac valve motions must handled very large displacements of valves in the fluid domain with surface contact aimed at preventing backward flows. 
In deformable vessels that experience at least $10 \%$ strain, fluid dynamics is strongly coupled to solid wall mechanics. In other words, the 3D Navier-Stokes equations are coupled to equations that describe large displacements of structural boundaries that have a viscoelastic behavior [43]. Hence, the fluid problem must be solved on a moving domain. Furthermore, lumped parameter models used to mimic physiological input and output impedances (multimodel coupling; e.g., [44]) cannot be directly coupled to a 3D detailled flow model to avoid spurious reflection phenomena; a 1D model for wave propogation must be interfaced.

As most breathing simulations are carried out in resting conditions (hence, walls are assumed rigid), most of the investigations on biofluid-biostructure interaction focus on blood flows. In this context, blood is flowing through a vessel made of a biological tissue of similar density; the coupling is strong and must be tackled very carefully to avoid numerical instabilities. Proper numerical schemes and algorithms must then be developed [45-50]. ${ }^{20}$

\subsubsection{Heat transfer in biological tissues}

Convective heat transfer by the blood supports the homeothermy of the human body. Blood circulation-associated heat exchangers enable heating or cooling of the body, depending on environment and body conditions. (During exercise, body temperature rises.) The main exchange surfaces (with a given thermal conductivity) are lungs and skin. Blood heat is transferred to the alveolus air (to be exhaled) and to the environmental air across the exchange tissues, i.e., the thin alveolocapillary membrane and the skin with its numerous cell layers. Heat exchangers can be bypassed. In particuler, limbs have two venous networks linked by anastomoses; the superficial venous network under the skin (a major heat exchanger) can be shunted in favor of the deep venous network that runs with main arteries.

Cancer treatments can use thermal ablation, ${ }^{21}$ which is less invasive than surgery. Therapy

\footnotetext{
${ }^{20}$ Fluid-structure interaction solvers developed in aeroelasticity, i.e., for coupled materials of quite different physical properties, cannot be used to solve blood flows in distensible (and collapsible) vessels.

${ }^{21}$ Image-guided radiofrequency ablation treats cancers particularly localized to the liver, kidney, and adrenal glands by heating. One or more radiofrequency needles are inserted into the tumor. Cryotherapy uses gas refrigerated cryoprobes, which are inserted inside the tumor, initiating the formation of ice balls to destroy cancerous cells by freezing and thawing processes. One of the main difficulties is the determination of the optimal position of the probes and treatment duration for complete destruction of cancerous cells without damaging too much surrounding normal cells. Another kind of tumor therapy consists of thermal and mechanical exposure to high-frequency focused ultrasound (HIFU). Tumor antigens and other compounds released from destroyed cells can stimulate the anti-tumor immunity. The optimal exposure time is an important parameter to avoid
} 
efficiency depends on the local blood flow. Heat transfer models use heat source(s) and sink(s) and effective conductivity to describe the thermal influence of blood flow. A continuum model cannot account for the local thermal impact of the vasculature, with countercurrent vessel segment pairs. The vasculature must indeed be modeled, down to a certain diameter, and be combined with a continuum model for heat transfer in the irrigated tissues.

The heat transfer coefficient $\left(h_{\mathrm{T}}\right)$ for the blood can be evaluated from the Sieder-Tate equation, when $q_{\mathrm{m}} c_{p} /\left(\mathcal{G}_{\mathrm{T}} L\right)>6$ :

$$
h_{\mathrm{T}} d_{h} / \mathcal{G}_{\mathrm{T}}=1.75\left(q_{\mathrm{m}} c_{p} /\left(\mathcal{G}_{\mathrm{T}} L\right)\right)^{1 / 3}\left(\mu_{\mathrm{w}} / \mu\right)^{0.14}
$$

where $d_{h}$ is the vessel bore, $L$ its length, $\mathcal{G}_{\mathrm{T}}$ the thermal conductivity, $q_{\mathrm{m}}$ the mass flow rate, and $\mu_{\mathrm{w}}$ the near-wall plasma viscosity at wall temperature.

\section{Pennes model}

The bioheat transfer equation proposed by Pennes in 1948 is used in physiological heat transfer modeling [51]. The Pennes model provides the rate of heat change in a given body tissue from the sum of the net heat conduction into the tissue, metabolic heat generation, and heating (or cooling) effects of the arterial supply. ${ }^{22}$ The blood flow-associated convective heat transfer term is given by: $q_{b} c_{b}\left(T-T_{a}\right)\left(q_{b}\right.$ : organ perfusion volumetric rate per unit volume of tissue; $c_{b}$ : specific heat of blood; $T_{a}$ : arterial temperature; and $T$ : local tissue temperature). The energy field in the perfused tissue domain is given by the bioheat transfer equation:

$$
\underbrace{\rho_{\mathrm{tis}} c_{\mathrm{tis}} \partial_{t} T}_{\text {time rate of internal energy change }}=\underbrace{\mathcal{G}_{\mathrm{Ttis}} \nabla^{2} T}_{\text {heat conduction }}+\underbrace{q_{b} c_{b}\left(T-T_{a}\right)}_{\text {perfusion effect }}+\underbrace{q_{\mathrm{met}}}_{\text {metabolism }}+\underbrace{q_{\mathrm{s}}}_{\text {source }},
$$

where $c_{\text {tis }}$ the specific heat of the explored biological tissue, $\rho_{\text {tis }}$ its density, $\mathcal{G}_{\text {Ttis }}$ its thermal conductivity, $q_{\text {met }}$ the metabolic heat source (rate of energy deposition per unit volume assumed to be homogeneously distributed throughout the tissue of interest, but usually

\footnotetext{
damage of normal cells, especially walls of neighboring blood vessels.

${ }^{22}$ Pennes underestimated the magnitudes of the conduction and convection terms in the energy balance, using inappropriate values of tissue thermal conductivity and tissue perfusion rate.
} 
neglected), and $q_{\mathrm{s}}$ a possible heat source (e.g., in the case of thermal ablation).

This equation is coupled to the equation of energy field in the flowing blood domain (Eq. 7), where $\rho=\rho_{b}, c_{p}=c_{\mathrm{b}}, \mathcal{G}_{\mathrm{T}}=\mathcal{G}_{\mathrm{b}}$ ( $\mathcal{G}_{\mathrm{b}}$ : perfusion conductivity), and $S=q_{h}$ (heat deposition due to an imposed source). The blood velocity (v) can be composed of two terms, a hemodynamic component and the so-called acoustic streaming ${ }^{23}$ generated by mechanical effects of high-frequency focused ultrasound when this therapeutic procedure is used to destroy a tumor.

\subsection{Blood circulation}

The blood circulation can be decomposed into 2 major compartments:

(1) microcirculation, at the tissular and cellular level, where the suspension of blood cell (strongly deformed or not) flows at low Reynolds number; and

(2) macrocirculation, in which blood can be supposed to be Newtonian in normal conditions and unsteadily flows at relatively high Reynolds number.

Two heart pumps combined into a single muscular organ beat synchronously to propel blood into the pulmonary and systemic circulation. The pulmonary and systemic blood volumes are shared between the arterial, capillary, and venous compartments.

The blood is conveyed from the drainage veins of the systemic circulation to the right atrium. It is then delivered into the right ventricle and expelled into the arteries of the pulmonary circulation for oxygenation into the lungs. The oxygenated blood is sent to the left atrium via the pulmonary veins. It then enters into the left ventricle to be propelled into the arteries of the systemic circulation, which distribute blood to every body organ

\footnotetext{
${ }^{23}$ Acoustic streaming describes non-linear, time-average fluid current near a vibrating surface (Rayleigh streaming) or the quasi-steady flow provoked by the passage of acoustic waves. An additional source of heat transfer results from forced convection induced by acoustic streaming. Rayleigh-Nyborg-Westervelt streaming theory relies on Navier-Stokes equations in which the inertia term is either neglected (creeping motion), i.e., at very low Reynolds numbers (Re $<1$ ) or not (Stuart streaming) [52]. In the present text, acoustic streaming is defined as a fluid motion caused by the absorption of the energy of high-frequency acoustic waves (generated by high-intensity ultrasounds) by the vibrating wall of large blood vessels and by the moving fluid that experience transverse (shear) waves of reduced amplitude.
} 
(including heart and lungs) for energy and nutrient supply and waste removal.

The macrocirculation includes large, mid-size, and small arteries as well as large, midsize, and small veins. The mechanical energy provided by the myocardium is converted into kinetic and potential energy associated with elastic artery distensibility, as well as into viscous dissipation. The bend is the basic geometrical element of the vasculature. In addition to curved segments, any branching can be assumed to be a bend juxtaposition, with a slip condition on the common boundary in a first approximation. However, flow separation occurs more easily in branchings than in curved vessels.

The microcirculation is composed of vessel of calibers from about five to few hundreds micrometers. The microcirculation that includes four main components - arterioles, capillaries, venules, and terminal lymphatic vessels — regulates blood flow distribution within organs, transcapillary exchanges, and removal of cell wastes.

The pressure difference between the entry of the arterial bed and exit of the veinous drainage drive the blood flow. Blood fills the cardiac cavities at a very low pressure and is propelled at high pressure. Atria and the set of atrioventricular and ventriculoarterial valves enable the pressure adaptation for ventricular filling and ejection. ${ }^{24}$

The pressure in the pulmonary circulation is much lower than that in the systemic circulation. Although the right pump is weaker than the left pump, flow rates are identical in both circulations, because the total pulmonary resistance is smaller than the total systemic resistance.

For efficient gas exchange between alveolar air renewed by inhaled air and pulmonary capillary blood loaded by carbon dioxide, ventilation flow must match the perfusion flow. However, a heterogeneous distribution of the perfusion in the lung is caused by the hydrostatic pressure difference between the lung apex (upper part) and basis (lower part) in the upright position. Therefore, the inhaled air is non-uniformly distributed to match the pulmonary perfusion.

\footnotetext{
${ }^{24}$ Atria can also act as auxiliary pumps that allow rapid ventricle filling at rest when the cardiac frequency is low.
} 


\subsubsection{Blood rheology}

Erythrocytes, or red blod cells (RBC), play a major role on the rheological behavior of the blood, as they represent $97 \%$ of blood cells. The blood thus behaves like a concentrated RBC suspension in a Newtonian solution (plasma), which is composed of ions and macromolecules interacting between them and bridging erythrocytes.

In large blood vessels (macroscale), the ratio between vessel bore and cell size is such that blood is considered as a continuous homogeneous medium. In capillaries (microscale), the blood is heterogeneous, transporting deformed cells in a Newtonian plasma. In the intermediate vascular compartment, flow is annular diphasic (rigid particle flow) with a core containing cells and a marginal plasma layer.

The rheological properties of non-Newtonian blood is dictated by the flow-dependent evolution of the blood internal microstructure, i.e., the state of the flowing cells (possible aggregation and deformation). The interactions between the conveyed plasma molecules and RBCs indirectly govern blood rheological behavior.

Shear-step experiments (steady state after a short transient regime) show that the blood has a shear-thinning behavior [53]. The shear-thinning behavior is exhibited by a pseudosigmoid relationship between the logarithm of the shear rate and the logarithm of the blood apparent viscosity (Fig. 1). This behavior is explained by RBC aggregation at low shear rates (high apparent viscosity values), whereas $\mathrm{RBC}$ deformation and orientation lead to a low viscosity plateau $\mu_{\infty}$ at high shear rates $\dot{\gamma}>10^{2} \mathrm{~s}^{-1}$ (Newtonian behavior range). A huge between-subject variability exists in the $\mu(\dot{\gamma})$ relationship. This variability points out the need for standardized procedure (temperature, $\mathrm{pH}, H t$, protein concentration, etc.). The $\mu(\dot{\gamma})$ relationship depends on $H t$ [54]. The very-low-shear-rate viscosity ( $\mu_{0}$; maximum of blood apparent viscosity, which is difficult to measure with available experimental devices, is about ten times greater than the high-shear-rate viscosity $\left(\mu_{\infty}\right)$. When both RBC aggregation and deformation are inhibited, the blood behaves almost like a Newtonian fluid [55].

However, blood is time-dependent shear-thinning fluid (thixotropy). Its behavior is affected by time-dependent, reversible changes in the blood structure. The thixotropic be- 


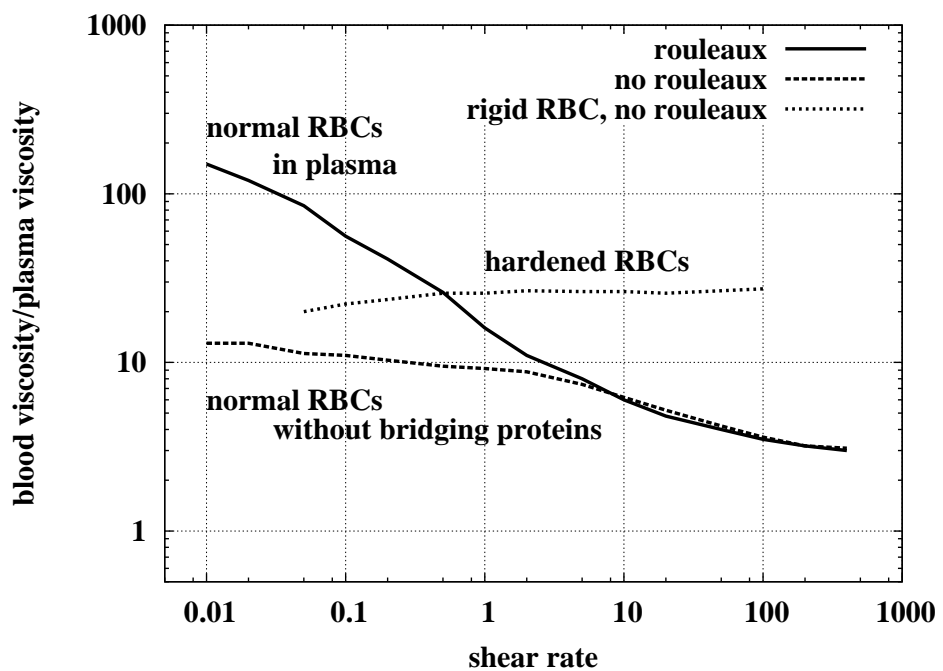

Figure 1: Relationship between the blood shear rate $\left(\mathrm{s}^{-1}\right)$ and the relative viscosity (bloodto-plasma viscosity ratio; from [53]). In static conditions, this relationship exhibit a shearthinning behavior. The relation depends upon the kinetic of formation and rupture of RBC aggregates at low shear rates and kinetics of $\mathrm{RBC}$ deformation and $\mathrm{RBC}$ orientation at high shear rates. Two ratios are involved: (1) the ratio between $\mathrm{RBC}$ size and flow length scale (vessel radius), and (2) the ratio between aggregation time constant and flow time scale (convection characteristic time for macrocirculation, transit time for microcirculation) in the explored vessel segment. Kinetics are governed by relaxation phenomena. Blood rheology is thus governed by variation in RBC suspension structure (thixotropic medium with viscoelastic behavior). The value at low shear rates is questionable because of the resolution limit of measurements. A between-subject variability exists, in particular in slope and curve inflection point values $\left(\dot{\gamma}_{(1 / 2)}\right)$.

havior is indeed explained by erythrocytic aggregation and desaggregation of erythrocytic rouleaux in red blood cells oriented in the streamwise direction. At rest, blood is composed of a rouleau network. Low shear rates deform and partially break the rouleau network into smaller rouleau networks and isolated rouleaux. At mid shear rates, only isolated rouleaux of various size are observed. At high shear rates, RBCs are scattered and oriented in the shear direction. A shear rate step induces a transient and stable strain regime, with a viscoelastic, elastothixotropic, and Newtonian response at low $\left(\dot{\gamma}=0.05 \mathrm{~s}^{-1}\right)$, mid $\left(\dot{\gamma}=1 \mathrm{~s}^{-1}\right)$, and high shear level $\left(\dot{\gamma}=20 \mathrm{~s}^{-1}\right)$, respectively [56].

Blood is viscoelastic material that experiences a loading history. ${ }^{25}$ Blood exhibits creep

\footnotetext{
${ }^{25}$ A loaded purely elastic body instantaneously deforms up to a given strain proportional to the applied force (it behaves as a spring). A loaded viscoelastic body progressively deforms up to an equilibrium state (as if a dashpot exists as a representative material component). Whereas elasticity is expressed in real parameters, viscoelasticity is described by frequency-dependent complex numbers.
} 
and stress relaxation during stress formation and relaxation [57]. Blood exposures to sinusoidal oscillations of constant amplitude at various frequencies reveal a strain-independent loss modulus and strain-dependent storage modulus. The existence of both moduli characterizes a viscoelastic material. The viscoelastic character can be estimated by the Weissenberg number, which is the product of the relaxation time scale and characteristic deformation rate.

The velocity profile in a pulsatile flow of a non-Newtonian fluid in a straight pipe is flat in the core. The shear rate $(\dot{\gamma})$ is then low in the core. If the fluid has a shear-thinning behavior, the fluid viscosity $(\mu)$ is then higher in the core than in the near-wall region where the shear rate is large.

Rheological data, which are provided by experimental results obtained in steady state conditions, ${ }^{26}$ are far from the physiological ones in the arteries [58]. Shear-step experiments do not match the blood flow dynamics. The flowing blood is characterized by a smaller convection time scale than the characteristic time of erythrocyte aggregation. In addition, erythrocytes expelled from the left ventricle, where they have been shaken during the isovolumetric contraction, do not have time to aggregate in the absence of stagnant blood regions. The blood, in large vessels, can then be considered to have a constant viscosity, greater than the dynamic viscosity of water and plasma (Table 7). On the other hand, non-Newtonian fluid flow must be simulated in stagnant blood regions (aneurysmal cavities and flow separation downstream from severe stenoses), when the residence time of erythrocytes is greater than the aggregation time constant.

In the framework of continuum mechanics, the time-dependent shear-thinning behavior and loading history of blood are incorporated via an appropriate formulation of the constitutive law. The most commonly used models for incompressible non-Newtonian fluid are the so-called generalized Newtonian models that are defined by the following constitutive equation:

$$
\mathbf{C}=-p \mathbf{I}+2 \mu \mathbf{D} ; \quad \mu=\mu(\dot{\gamma}) ; \quad \dot{\gamma}=(2 \mathbf{D}: \mathbf{D})^{1 / 2}
$$

\footnotetext{
${ }^{26}$ Blood behavior depends on the structure of the RBC population.
} 
Table 7: Blood $\left(H t=45 \%, T=37^{\circ} \mathrm{C}\right)$ and water $\left(T=37^{\circ} \mathrm{C}\right)$ physical properties (Source: [2]).

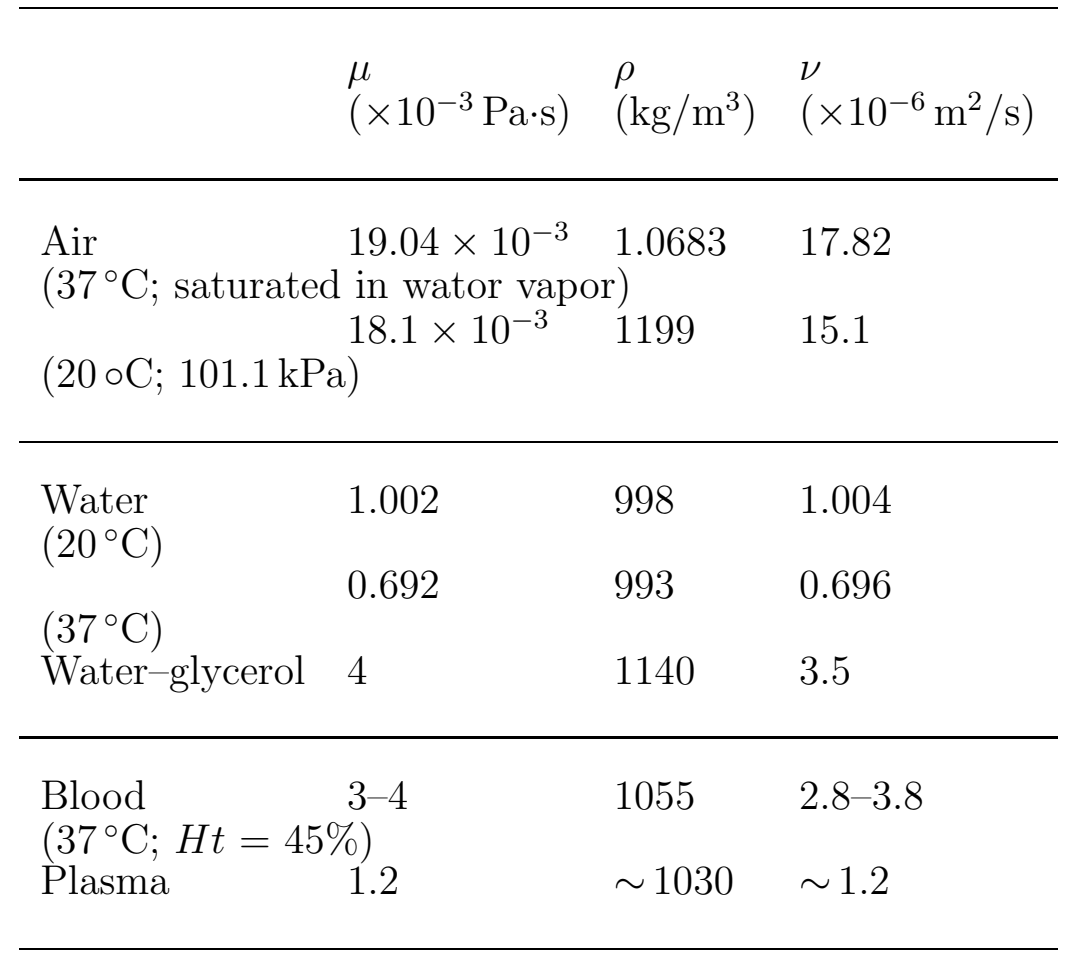

The power law and the Casson, Herschel-Buckley, Quemada, and Carreau models belong to the family of generalized Newtonian models. The Carreau model is given by:

$$
\frac{\mu(\dot{\gamma})-\mu_{\infty}}{\mu_{0}-\mu_{\infty}}=\left(1+\left(\dot{\gamma}_{(1 / 2)} \dot{\gamma}\right)^{a}\right)^{\frac{n-1}{a}}
$$

where $\dot{\gamma}_{(1 / 2)}$ is the shear rate at mid-slope. It is easily implementable in fluid solvers for numerical simulations. ${ }^{27}$ It can be used in steady flows, as it captures adequately the shearthinning behavior. ${ }^{28}$

Generalized Newtonian models used to mimic the shear-thinning blood behavior do not take into account the stress and strain history of blood and aggregation-desaggregation cycles of red blood cells. They are not relevant in pulsatile flows.

\footnotetext{
${ }^{27}$ Power values are obtained by fitting the power-law region of the shear-thinning behavior.

${ }^{28} \mathrm{~A}$ value set has been proposed [58]: $a=2, \mu_{0} \sim 40 \mathrm{mPa} \cdot \mathrm{s}, \mu_{\infty} \sim 4 \mathrm{mPa} \cdot \mathrm{s}, \dot{\gamma}_{(1 / 2)} \sim 0.25 \mathrm{~s}^{-1}, n \sim 1 / 3$. This set of values fit the experimental data carried out in steady state and are thus irrelevant for pulsatile flows.
} 
In stagnant flow region, in particular when an aneurysm develops in the arterial bed with a configuration that promotes a local flow stagnation, red blood cells can have time to aggregate and form rouleaux. These rouleaux can be modeled as purely elastic dumbbells, the length of which change as rouleaux aggregate and fragment. The resulting suspension of isolated erythrocytes and erythrocytic rouleaux oriented in the streamwise direction constitutes an incompressible, shear-thinning, viscoelastic, and thixotropic fluid. The set of equations, which is similar to an Oldroyd model, to solve becomes [59]:

$$
\begin{aligned}
\rho D_{t} \mathbf{v} & =\boldsymbol{\nabla} \cdot \mathbf{C}+\boldsymbol{\nabla} \cdot \mathbf{E}, \\
\boldsymbol{\nabla} \cdot \mathbf{v} & =0, \\
2 \mu \mathbf{D} & =\mathbf{E}+\tau\left(D_{t} \mathbf{E}-\left[\boldsymbol{\nabla} \mathbf{v} \cdot \mathbf{E}+\mathbf{E} \cdot(\boldsymbol{\nabla} \mathbf{v})^{T}\right]\right), \\
D_{t} \overline{\mathbf{N}} & =-\frac{1}{2} \mathrm{~F}(\mathbf{D})\left(\overline{\mathbf{N}}-\overline{\mathbf{N}}_{s t}\right)\left(\overline{\mathbf{N}}+\overline{\mathbf{N}}_{s t}-1\right),
\end{aligned}
$$

where $\mathbf{E}$ represents the contribution of the elastic dumbbells to the total Cauchy stress, $\overline{\mathrm{N}}$ the average size of rouleaux, $\mu=H t(\operatorname{Bo} T+\kappa) \tau$ the polymer viscosity $(H t$ : red blood cell concentration, Bo: Boltzmann constant, $T$ : absolute temperature, $\tau(\overline{\mathrm{N}}, \mathbf{D})$ : relaxation time that depends on erythrocyte aggregation and fragmentation rates and relaxation time of a single particle), $F\left(\mathbf{D}, \overline{\mathbf{N}}_{s t}\right)$ the erythrocytic rouleau fragmentation rate, and $\overline{\mathbf{N}}_{s t}(\mathbf{D})$ the value of average aggregate size $\mathrm{N}$ in a simple shear, steady flow with shear rate $\mathbf{D} .^{29}$

In the elastic stress component due to aggregates of all sizes (various aggregated cell number $[\mathrm{N}]$ ), the relaxation time $\tau_{\mathrm{N}_{i}}$ for aggregates of size $\mathrm{N}_{i}$ is replaced by an average value $\tau_{\overline{\mathrm{N}}}$

\subsubsection{Hemolysis modeling}

Ventricular assist devices, artificial cardiac valves, venous filters, and catheters yields unusual impact surfaces that can damage erythrocyte membranes, thereby causing hemolysis. In addition, subsequent release of hemoglobin in the plasma may provoke renal failure due to free hemoglobin toxicity. Hemolysis depends on the magnitude, duration, and nature of the

\footnotetext{
${ }^{29} \overline{\mathbf{N}}_{s t}=f\left(\mu_{0} / \mu_{\infty}, \mathbf{D}, \mathbf{A}(\mathbf{D}), \tau_{\infty}\right)$, where $\mathbf{A}(\mathbf{D})$ is the aggregation rate and $\tau_{\infty}$ the relaxation time when the shear rate tends to infinity. The ratio $\mu_{0} / \mu_{\infty}=\left(1+\left(\mu_{0} / \mu_{\infty}\right) \dot{\gamma}\right) /(1+\dot{\gamma})$.
} 
local stress field.

According to [60], "up to now, neither the exact mechanisms of blood damage nor the tolerable shear loads have been explicitly defined". Hence, erythrocyte damage is commonly assessed by a correlation between the shear stress and exposure time. The higher the shear stress and the longer the residence time of erythrocytes, the greater the risk of damage. An analysis based on the volumetric residence time related to the number of particles in the computational cell at a given time, the volume of the cell, the hemolysis rate, and the number of erythrocytes entering the computational cell was proposed by [61]. On the other hand, the residence time has been considered as simply the ratio of the distance between neighboring nodes divided by the in-cell averaged flow velocity [62]. Erroneous predictions and computational problems may arise when this velocity becomes null. For complex geometries, these approaches are prone to error since they are sensitive to the distribution of particles or streamlines in the computational domain. Moreover, this operation is time-consuming, depends strongly on a detailed knowledge of the flow topology inside the device.

To evaluate the blood damage from medical devices such as ventricular assist devices with continuous blood flow, ASTM F1841-97 standard [63] proposes three relations for the laboratory measurement of the erythrocyte damage: the normalized index of hemolysis (NIH), normalized milligram index of hemolysis (mgNIH), and modified index of hemolysis (MIH). The first two indices measure the increase in plasma-free hemoglobin in $\mathrm{g}$ and $\mathrm{mg} / 100 \mathrm{l}$ of pumped blood, respectively. The last index measures the increase in plasma-free hemoglobin, normalized by the total quantity of hemoglobin in the volume of blood pumped by the device in a closed circuit. Hence, the unit-free MIH is a preferred index.

A numerical method has then be proposed for the prediction of the NIH and the MIH for the preselection and design optimization of implantable devices [64]. This methodology does not require particle-seeding, streamlines, or residence time computation, thus avoiding related problems. It directly computes the hemolysis indices from the velocity and stress fields. Since the procedure is not discrete (as opposed to the previously mentioned streamline or particle approaches), its accuracy can be measured and embedded within standard grid convergence analysis. Finally, the procedure is cost-effective and requires simple evaluations 
at the computational cell level as a post-treatment of numerical solutions of the NavierStokes equations. The premises of the hemolysis model based on the Giersiepen et al. correlation [65] are shared by others [61, 62].

In steady state, when the damage is too high, i.e., the experiment duration remains short enough, the equation set to be solved is given by:

$$
\left\{\begin{aligned}
\mathrm{d} & =\left(\left[3.62 \times 10^{-7}\right] \times C_{V M}^{2.416}\right)^{\frac{1}{0.785}} \\
\mathrm{D} & =\left(\int_{V} \mathrm{~d} d V / q_{v}\right)^{0.785} \\
N I H & =H b \times \mathrm{D} \times 100 \\
M I H & =\mathrm{D} \times 10^{6}
\end{aligned}\right.
$$

where $\mathrm{d}$ is the local degradation rate, $H b$ the total hemoglobin concentration and $C_{V M}$ the Von Mises stress according to the recommendation given in [66]. This hemolysis model is adequate only when the flow is steady such as in the case of continuous cardiac assist pump with a single outlet. The result must be considered more qualitatively than quantitatively for a classification purpose of the resulting damage caused by a given implantable device. ${ }^{30}$ The computation of the damage rate $\mathrm{D}$ requires to solve a hyperbolic partial differential equation.

\subsubsection{Microcirculation}

Arterioles are small precapillary resistance vessels. They are richly innervated by sympathetic adrenergic fibers and highly responsive to vasoconstriction signals from the sympathetic nervous system. They constitute a major site for the regulation of systemic vascular resistance. The primary function is flow regulation, thereby determining nutrient delivery and catabolite washout. They partially regulate capillary hydrostatic pressure and fluid exchanges. In addition, a low ambient temperature causes a constriction of skin arterioles to reduce heat loss. In some organs, precapillary sphincters can regulate the number of perfused capillaries. Venules are collecting vessels. Sympathetic innervation of larger venules

\footnotetext{
${ }^{30}$ All hemolysis models are not asymptotically consistent. Prediction higher than $100 \%$ damage can be obtained. A version asymptotically correct was derived in [67].
} 
influences the contraction state of venular walls, thereby controlling the flow in capillaries and contributing also to the regulation of capillary hydrostatic pressure and blood-tissue exchanges.

Lymph has a composition similar to plasma, but the protein concentration in the lymph is about half that of plasma. Lymphatic vessels participate in tissue clearance of molecules, cell removal after inflammation, and interstitial fluid homeostasis. Lymphatic valves prevent lymph back flow, thus promoting lymph flow into the systemic circulation via the thoracic duct and subclavian veins. Lymph flow is very slow (Stokes flow). Large lymphatic vessels have muscular walls that support a local control of the lymph circulation. Spontaneous (stretch-dependent) vasomotion, i.e., oscillations of vessel mural tone, in terminal lymphatic vessels fosters lymph transport. ${ }^{31}$ In addition, sympathetic nerves cause contraction of the lymphatic smooth muscle.

The capillary blood circulation is also a Stokes flow. The short distance between the capillary lumen and tissue cells is adapted to molecular exchanges. Capillaries indeed are the primary site of exchange for fluid, electrolytes, gases, and macromolecules, mainly by filtration, absorption, and diffusion. Fluid can move from the intravascular compartment to the extravascular milieu composed of cellular, interstitial, and lymphatic subcompartments. The transport of fluid and solutes (electrolytes and small molecules) is determined by hydrostatic and osmotic transendothelial pressures, as well as endothelium permeability. Fenestrated capillaries have the highest permeability. In most capillaries, filtration of fluid exceeds reabsorption by the capillary endothelium. Excess fluid within the interstitium is removed by the lymphatic system.

The capillary flow locally depends on the upstream resistance in the arterioles and the downstream resistance in the venules. Local control of substance transport to the tissue is done by:

(1) recruitment ${ }^{32}$ of terminal arterioles (the higher the number of open capillaries, the greater the solute delivery and waste removal);

\footnotetext{
${ }^{31}$ Smooth muscle cells of the stretched lymph vessels rhythmically contract at low frequency due to lymph accumulation.

${ }^{32}$ Blood vessel recruitment can refer to angiogenesis that ensures normal tissue growth. Here, blood vessel recruitment corresponds to the contribution of previously unperfused capillaries to increase the total surface area of the mass exchange between the blood and the perfused tissue, thereby maintaining a proper oxygenation when the metabolic demand increases.
} 
(2) autoregulation (maintenance of a constant flow despite changing vascular pressure) in the arteriolar compartment and vasodilation resulting from increased local metabolism; and

(3) vascular permeability.

\section{Quasi-steady flow}

In the microcirculation, both the Reynolds and Stokes numbers become much smaller than one. ${ }^{33}$ Centrifugal forces do not significantly affect the flow in this vascular compartment, where the motion is quasi-independent on the vessel geometry.

Capillaries have walls that are very thin. A single layer of endothelial cells over a basement membrane represent the major barrier of the exchange zone. In this exchange region, where blood velocity is low, the quantity of interest is the transit time of conveyed molecules and cells. The mean residence time is the ratio of capillary volume to the local flow rate.

\section{Particle flow}

In microcirculation, blood flow becomes heterogeneous and a particle flow must then be considered. The particles are mainly the deformable blood cells, primarily erythrocytes. The particle concentration is such that interparticle distance is greater than particle size. Between-cell interactions are then lower than fluid-cell interactions. Solid particle flows depend on flow characteristics and particle features (shape, size, concentration, deformability, and buoyancy) with respect to the suspending fluid (plasma).

In small blood vessels, where inertia is negligible, a tiny lubrication film between the endothelium and flowing cell membrane favors motion of the deformed cells. Compared with a homogeneous Newtonian fluid in the same flow conditions, velocity is reduced and resistance is augmented.

The plasma peripheral layer explains the plasma skimming. This process refers to blood

\footnotetext{
${ }^{33}$ In the microcirculation, the blood velocity is of order $\mathcal{O}\left(10^{-2}\right)$ to $\mathcal{O}\left(10^{-3} \mathrm{~m} / \mathrm{s}\right)$ and the vessel radius of $\mathcal{O}\left(10^{-5} \mathrm{~m}\right)$. Hence, the Reynolds and Stokes number are much less than one.
} 
cell segregation at branch points of the capillary bed that generate a tiny side branch and a capillary of usual size. As blood cells flows in the core of the microvessel lumen, these cells flow in the broader capillary, whereas plasma close to the parent vessel wall supplies the very narrow side branch. This phenomenon can be observed particularly in kidneys, where blood filtration occurs.

In arterioles, flow-seeded particles steadily conveyed in a vessel migrate transversally due to inertia $[68,69]$. Transverse migration of the seeded particles depends on various factors: inertial lift, local gradient in shear rate, particle volume fraction, wall repulsion, and deformation of flexible particles [70, 71].

The arteriolar flow is characterized by the Fahraeus effect (local hematocrit $H t<$ global $H t$ ) and the Fahraeus-Lindqvist effect, i.e., blood viscosity dependence on the vessel caliber. In the microcirculation, the hematocrit (the fraction of erythrocyte volume to the blood volume) decreases due to a higher plasma fraction for a given blood volume. This phenomenon reduces the viscous dissipation with respect to a suspension of a higher cell concentration in small vessels. The Fahraeus-Lindqvist effect can be explained by the interaction of the concentrated suspension of deformable erythrocytes with the vessel wall [72]. The erythrocytes are located in the flow core region, whereas a cell-free layer exists near the wall.

Any blood cell pass through narrow capillaries owing to their deformability. Highly deformable erythrocytes are capsules, i.e., essentially concentrated suspensions of hemoglobin enclosed by elastic membranes. The behavior of a deformed capsule in a shear flow strongly depends on the unstressed shape and viscosity of its internal fluid relative to the solvent's viscosity. Rheological properties of the membrane control resistance to strain of the capsule under applied stresses. The magnitude of the deformation depends on the rheological properties of the cell membrane that controls the resistance of the capsule to applied stresses and on the magnitude of the tangential and normal components of applied stresses. 


\section{Mass exchange}

Solute can be transported by either diffusion due to a concentration gradient and convection due to a hydrostatic pressure difference between the vessel lumen and the surrounding tissues. Nutrients and metabolic wastes are transported between blood and tissue cells by convection and diffusion facilitated by a high residence time in the capillary.

Diffusion from a compartment of higher concentration to a region of lower concentration across a membrane of infinitesimal thickness is governed by the Fick law. Brownian motion states that substance flux is proportional to the solute permeability coefficient, which depends on diffusion coefficient and solute solubility, transport surface area and concentration gradient.

The hydraulic conductivity measures the porosity of the capillary wall for the water flux. The filtration coefficient is the hydraulic conductance per unit exchange surface area.

The net driving pressure for water motion is determined by the sum of the hydrostatic and osmotic contributions. Water motion into or out of the capillary, across the endothelium, is partly driven by osmosis. Water moves from regions of low-solute concentration to regions of higher-solute concentration. Osmotic pressure is the pressure required to stop the net flow of water across a membrane such as a cell layer that separates solutions of different composition. The osmotic pressure $\Pi$ thus depends on the vessel wall structure. An ultrafiltrate results from the material separation by a semipermeable wall under a given filtration pressure. Suspended solids and solutes of high molecular weight are retained, whereas water and low molecular weight solutes cross the wall.

The van't Hoff equation describes the osmotic pressure on one side of the membrane. It is valid for a single chemical species of concentration $c$ : $\Pi=R_{\mathrm{g}} T c$, where $R_{\mathrm{g}}$ is the gas constant and $T$ the temperature. A given solution can lead to different osmotic pressures when the filtration membrane porosity differs. The osmotic pressure difference must be multiplied by a reflection coefficient associated with capillary permeability to the proteins responsible for the osmotic pressure. The reflection coefficient, or selectivity coefficient, measures the probability of solute penetration across the vessel wall. A reflection coefficient 
equal to 1 means that the vessel wall is impermeable to the molecule (most of the proteins are responsible for the osmotic pressure); a coefficient equal to 0 means that there is no transport restriction, such as for small solutes (e.g., salts and glucose). In the range bounded by these two values, the wall layer is semipermeable to solute with some amount that does not penetrate (reflected off) the barrier.

Materials that can cross the vessel wall include respiratory gases, water, ions, amino acids, carbohydrates, proteins, lipid particles, and cells. The capillary permeability is high for water and moderate for ions, lipids, and proteins. Fluid motion between blood and tissue fluid is mainly determined by two opposing forces, hydrostatic and osmotic pressure differences between blood and tissue fluid. Water flux results from the imbalance between hydrostatic and osmotic pressures, for given features of the vessel wall layer and exchange surface area. A net motion of water out of (positive water flux) or into (negative water flux) the vessel leads to filtration and absorption, respectively.

Consider a capillary in which the intraluminal pressure drop $\Delta p_{i}$ is linear and the tissue pressure $p_{w}$ constant. The capillary wall is semipermeable. The solvant transport due to $\Delta p=p_{i}-p_{w}$ is decreased by $\Delta \Pi$ due to the presence of macromolecules in the capillary lumen, which do not cross the wall. When the local effective pressure associated with osmotic and hydrostatic pressure differences between the lumen and the wall (subscript lw) $p_{\text {eff }}=\Delta p_{\mathrm{lw}}-\Delta \Pi_{\mathrm{lw}}>0$, the plasma is filtrated. When $p_{\text {eff }}<0$, the fluid is reabsorbed from the interstitial fluid into the capillary.

The capillary hydrostatic pressure is normally much greater than the tissue hydrostatic pressure in the upstream capillary segment. The resulting hydrostatic pressure gradient drives water out of the capillary lumen into the interstitium. The ultrafiltrate that results from semipermeable wall filtration has the same ion concentration as plasma and does not contain large molecules. The plasma osmotic pressure is usually much greater than the interstitial osmotic pressure in the downstream capillary segment. The osmotic pressure gradient across the capillary supports water reabsorption from the interstitium into the capillary. A fraction of filtrated plasma is sucked back from the interstitial liquid into capillaries and the remaining part is drained by lymphatic circulation into large veins. In 
summary, plasma is filtered out of the capillary entry segment and reabsorbed back into the capillary exit segment. In normal conditions, the filtration rate is equal to the sum of the reabsorption rate and lymphatic flow rate, thereby avoiding edema.

Solute crosses easily the vessel wall through gaps of fenestrated capillaries. In microvessels with continuous endothelium, the main route for water and solutes is intercellular clefts in the capillary endothelium, except when strong tight junctions exist, such as in the brain capillaries, where they define the blood-brain barrier. Tight junctions are composed of a branching network of sealing independent strands formed from rows of transmembrane proteins. In other body's organs, plasma and interstitial fluid are similar fluids that communicate through intercellular clefts. A typical endothelial cleft is a passage between two apposed endothelial cells that contains one or more tight junctions interspersed with wide zones of uniform width. The tight junctional region is discontinuous along the axial length of the capillary and contains a separation of $4 \mathrm{~nm}$ at the point of apposition of the plasma membranes of adjacent cells.

Transcapillary transfer of water and solutes in both continuous and fenestrated endothelia was described in terms of three parallel routes: a water pathway across the endothelial cells; a set of small pores (caliber 4-5 nm); and a population of larger pores (bore 20-30 nm) [73].

In addition, moving chemical species must cross the glycocalyx. The glycocalyx refers to an extracellular polymeric material made of glycoproteins and proteoglycans anchored to the apical (wetted) part of the plasma membrane. A thin matrix at the cleft entrance can indeed be a major determinant of the permeability of the capillary wall. The associated fiber matrix model of capillary permeability associated with the endothelium glycocalyx (thickness $\sim 100 \mathrm{~nm}$; fiber spacing of $7 \mathrm{~nm}$ ) provides a basis for molecular size selectivity, especially in a fenestrated endothelium. A fiber matrix model can also be applied for the cleft itself, because clefts contain matrix components. Models have predicted that the fiber layer (typical thickness $100 \mathrm{~nm}$ ), which extends from the endothelium surface into the cleft entrance region, sieves solutes [74].

The estimated between-cell exchange area is on the order of $0.4 \%$ of the total capillary surface area. The pore theory simulates the cleft passages between adjacent endothelial 
cells in continuous endothelium. The array of junctional strands between endothelial cells is indeed interrupted at intervals, allowing water and solute fluxes. The pore density is defined by the effective fraction of the cleft width used for molecular exchange. The pore size is determined by the interfiber spacing in a fiber matrix at the cleft entrance. The whole membrane permeability coefficient is the sum of the different route coefficients. The membrane reflection coefficient is the sum of the individual coefficients of the parallel paths weighted by the fractional contribution of each path to membrane hydraulic conductivity. Despite its limitations, ${ }^{34}$ the pore theory is a useful pedagogical tool. Moreover, any fiber matrix-based modeling must not only take into account fiber size and volume, but also matrix organization [73].

The effective pore radius, a selectivity measure, depends on interstices in the fiber matrix, i.e., on matrix composition and arrangement. The effective pore number depends on the size and frequency of endothelium passages, in the intercellular spaces as well as through the cells. Water and small solutes can indeed cross the endothelial cells, using specific channels. For example, aquaporins are membrane water-transport proteins [75]. Aquaporin-1 is found in endothelia. However, the contribution of the transcellular transfer to the net flux is usually supposed to be either small or negligible. In addition, elevated transmural pressures on endothelial cell culture on rigid, porous supports increase the endothelial hydraulic conductivity [76]. It is postulated that elevated shear stresses in endothelial clefts caused by increased transmural flow heightens the hydraulic conductivity.

Macromolecules can cross the endothelium between the cells (paracellular transport) or essentially through endothelial cells (transcytosis), using receptors, specific or not, and vesicles. Microvascular wall models have been proposed with pores for small and intermediate-sized molecules and transendothelial channels for macromolecules. However, the macromolecule transport, which is convection-independent, needs endothelial vesicles. Vacuole-like structures have been observed, isolated inside the endothelial cell or from luminal membrane invaginations, or connected to the abluminal compartment [77]. The luminal and abluminal (opposite surface in contact with the extracellular matrix) surfaces of the capillary endothelium are dynamical, with invaginations and protrusions associated with environmen-

\footnotetext{
${ }^{34}$ Macromolecular transport is not always coupled to water flows. Furthermore, there are capillaries with no large pores.
} 
tal stimuli. Moreover, vesicle translocation between luminal and abluminal membranes is accelerated when the transendothelial pressure rises. Endothelium permeability can increase due to the existence of vesiculovacuolar organelles without formation of trans- or intercellular gaps.

The fluid volume flux $(J)$, or volumetric flow rate per unit area, across the endothelial barrier, which is modeled by a membrane, is given by the Starling relationship:

$$
J=\mathcal{G}_{\mathrm{h}}\left(\Delta p-\kappa_{\mathrm{o}} \Delta \Pi\right)
$$

where $\mathcal{G}_{\mathrm{h}}$ is the hydraulic conductivity, $\Delta p=p_{l}-p_{\text {int }}$ the difference in hydrostatic pressures between capillary lumen $\left(p_{l}\right)$ and interstitial fluid $\left(p_{\text {int }}\right), \Delta \Pi=\Pi_{l}-\Pi_{\text {int }}$ the difference in osmotic (oncotic) pressures between capillary lumen $\left(\Pi_{l}\right)$ and interstitial fluid $\left(\Pi_{i n t}\right)$, and $\kappa_{\mathrm{o}}$ the osmotic reflection coefficient of vessel wall.

Kedem-Katchalsky equations incorporate convection and diffusion:

$$
\left\{\begin{array}{l}
J_{\mathrm{w}}=\mathcal{G}_{\mathrm{h}}\left(\Delta p-\kappa_{\mathrm{o}} \Delta \Pi\right), \\
J_{\mathrm{s}}=\mathcal{P} \Delta c+J_{\mathrm{w}}\left(1-\kappa_{\mathrm{d}}\right) c_{*},
\end{array}\right.
$$

where subscripts w and s stand for water and solute, respectively, (the main solute is albumin) $\mathcal{P}$ the membrane permeability for the solute, $\Delta c$ the difference in solute concentration across the endothelium, $c_{*}$ the averaged solute concentration within a pore that represents an endothelial cleft, and $\kappa_{\mathrm{d}}$ the solute drag reflection coefficient that depends on membrane permeability for both water and solute.

\section{Local regulation}

The wetted surface of any segment or organ of the cardiovascular system is covered by the endothelium, which constitutes the interface between the flowing blood and the deformable solid wall. Endothelial cells detect hemodynamic stresses via mechanosensors. Blood vessel walls quickly react to loads applied to it by the flowing blood. In any segment of a blood 
vessel, endothelial and smooth muscle cells sense the space and time variations (high amplitude) in wall shear stress (small magnitude) and wall stretch (large magnitude) generated by the blood pressure, respectively. These cells respond within a relatively short time scale (for biological responses, i.e., from seconds to hours) to adapt the vessel caliber according to the loading, especially when changes exceed the limits of the usual stress range. This regulatory mechanism is much swifter than nervous and hormonal control. The mechanotransduction pathways determine the local vasomotor tone and subsequently the lumen bore of the reacting blood vessel $[67,78,79]$.

Mechanotransduction is the process that starts with the detection of mechanical stresses and its translation into chemical signals at the plasma membrane. This translation comprises a chain of chemical reactions that are controlled both in time and space. Transduction begins at the cell cortex, where signaling effectors are recruited. Certain signaling mediators are transported by the cell cytoskeleton from the cytoplasm to the nucleoplasm to trigger adequate gene expression and protein synthesis. The cytoskeleton itself transmits mechanical constraints and reacts to the stress field to adapt the cell shape. Regulators are newly synthesized or stored in vesicles and released in the extracellular matrix to carry out their auto-, juxta-, para-, and eventually endocrine functions.

Three types of mechanotransductions exist according to the compartment of the arterial bed: large and small resistive arteries and arterioles (Table 8). In large arteries, the regulation of the vasomotor tone aims at correcting high or low pressure to keep the cardiac postload at normal levels. When the pressure increases, the response is a vasodilation to reduce local resistance. In small arteries and arterioles, the goal of the autoregulation is to maintain the flow rate constant. An increase in pressure in a given restricted range thus causes an additional rise in pressure as flow is the ratio between pressure and resistance. In small tissue-perfusing vessels, the caliber is controlled by the local energy demand. The higher the local cell activity, the larger the vessel bore. The vasodilation must be associated with an increase of cardiac output by nervous and endocrine control of the cardiac function to ensure an appropriate perfusion. 
Table 8: Mechanotransduction types and compartments of the arterial network.

\begin{tabular}{lll}
\hline $\begin{array}{l}\text { Arterial } \\
\text { compartment }\end{array}$ & Function & Goal \\
$\begin{array}{l}\text { Large } \\
\text { arteries }\end{array}$ & $\begin{array}{l}\text { Body's territory } \\
\text { irrigation }\end{array}$ & $\begin{array}{l}\text { Regulation of } \\
\text { cardiac postload }\end{array}$ \\
$\begin{array}{l}\text { Small resistive } \\
\text { arteries }\end{array}$ & $\begin{array}{l}\text { Organ } \\
\text { perfusion }\end{array}$ & $\begin{array}{l}\text { Maintenance of a } \\
\text { constant flow rate }\end{array}$ \\
\hline Arterioles & $\begin{array}{l}\text { Tissue } \\
\text { perfusion }\end{array}$ & $\begin{array}{l}\text { Vasodilation caused } \\
\text { by increased local metabolism }\end{array}$ \\
\hline
\end{tabular}

\subsection{Respiration}

The main function of the respiratory system is to convey respiratory gases from the atmosphere to the gas exchange region and vice versa using the respiratory pump mainly made of respiratory muscles of the thoracic cage under the control of the nervous system. Molecules of oxygen and carbon dioxide are transported by diffusion through the alveolocapillary membrane between alveoli and blood capillaries.

At rest, inhalation results from decrease of intrathoracic pressure associated with the deformation of the chest walls initiated by the diaphragm and supported by the external intercostal muscles to inflate airways and alveoli, in which the pressure becomes lower than that of the atmosphere. The following exhalation is passive, using elastic recoil forces developed during inspiratory stretch. Forced inspiration and expiration require the intervention of additional muscles.

\subsubsection{Architecture of the respiratory tract}

The respiratory tract is a duct network made of serial compartments. It begins from its apertures to ambiant air, i.e., openings of the nose (nostrils) and mouth (interlabial space) with the upper airways. The latter include the nasal or buccal cavity in some circumstances, e.g., exercise, lung function testing, and diving with breathing apparatus, pharynx, and larynx. Usually, the nose with its associated sinuses is the proximal airway segment. It serves as entry and exit segment during inspiration and expiration, respectively. After the 


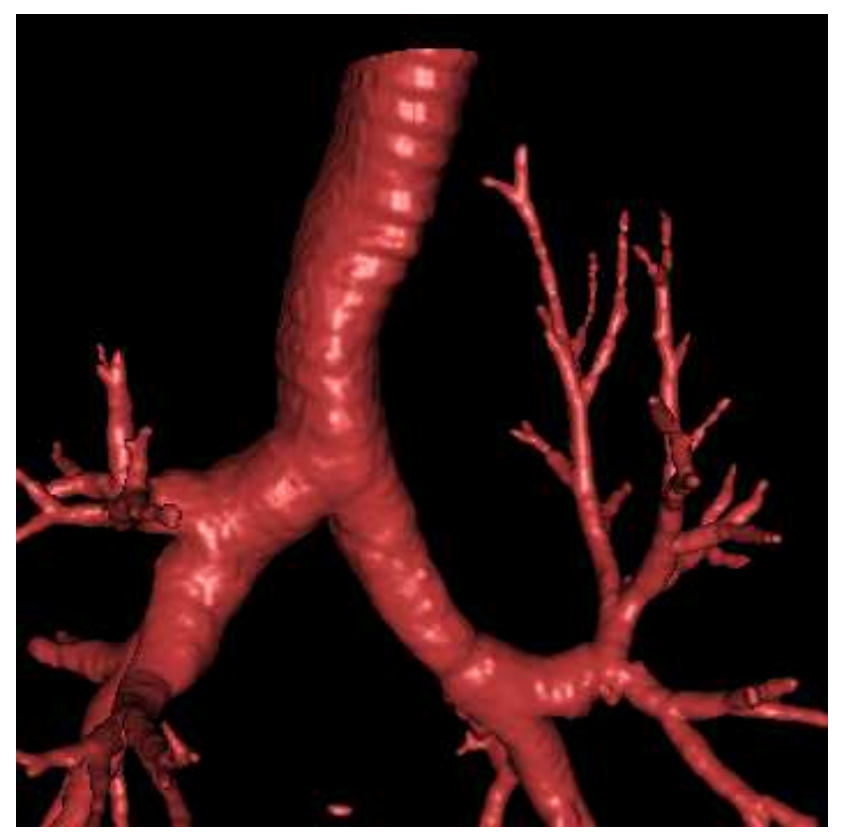

Figure 2: Three-dimensional reconstruction of the proximal part of the tracheobronchial tree. Images processing allows to extract a tree down to generation 6 to 7 (courtesy C. Fetita).

larynx characterized by a deformable nozzle, the trachea possesses an extra- and intrathoracic segment. In the thorax, the trachea bifurcates to generate two main, or stem bronchi. Each stem bronchi penetrates within the lung and gives rise to a bronchial tree. Intraparenchymal proximal airways comprise lobar and segmental bronchi. Distal airways encompass lobular bronchi and bronchioles. The most distal part pertains to the pulmonary acinus, the site of gas exchange. The respiratory tract is characterized by two highly irrigated terminal regions — nose and alveoli — that can serve for non-invasive drug uptake.

Each bronchial tree, is composed of successive generations of airways (Fig. 2). The greater the duct generation level, the shorter and the narrower the respiratory conduit, and the higher their number. From the trachea, the tracheobronchial tree divides into mostly paired branches of unequal length and diameter (irregular dichotomy). The maximum number of branches varies according to the path length inside the lung parenchyma, the number of bronchial divisions ranging from 17 to 26 .

Daughter bronchi usually arise from asymmetric bifurcations and, sometimes, trifurcations (terminal branchings). Lateral branchings (a small bronchus branches off on the edge 
Table 9: Geometrical data of the tracheobronchial tree. Airway dimensions depend on age, lung size, and lung inflation degree $\left(A_{t}, d_{t}, A_{b}, d_{b}\right.$ : cross sectional area and caliber of a trunk (parent bronchus) and of a branch).

\begin{tabular}{ll}
\hline $\begin{array}{l}\text { Geometrical } \\
\text { parameters }\end{array}$ & Value \\
\hline$L / d_{t}$ & $\sim 3.5$ \\
$L / d_{b}$ & $\sim 3.5$ \\
$d_{b} / d_{t}$ & $0.7-0.8$ \\
$(\mathrm{G} 0-\mathrm{G} 16)$ & \\
$2 A_{b} / A_{t}$ & $1.20-1.25$ \\
Branching angle & $64-100$ degrees \\
$R_{c}$ & $5-10 d_{t}$ \\
\hline
\end{tabular}

Table 10: Generation bore and length in the tracheobronchial tree for a respiratory path with a large number of respiratory pipe generations.

\begin{tabular}{llll}
\hline Generation & $\begin{array}{l}d_{h} \\
(\mathrm{~mm})\end{array}$ & $\begin{array}{l}L \\
(\mathrm{~mm})\end{array}$ & $L / d_{h}$ \\
\hline 0 & $15-22$ & $100-120$ & $6-7.5$ \\
1 & $10-15$ & $30-50$ & $3-4$ \\
2 & $7-11$ & $15-25$ & 2 \\
$3-8$ & $4.5-8$ & $10-15$ & $1.4-2.2$ \\
$8-17$ & $2-3.5$ & $6.5-9.5$ & $2.7-3.2$ \\
$17-23$ & $1-2$ & $3-6$ & 3 \\
$23-35$ & $0.8-0.2$ & $0.5-1$ & $0.6-1.5$ \\
\hline
\end{tabular}

of a much larger bronchus) can be observed. The area ratio, i.e. the ratio between the cross-sectional area $\left(A_{t}\right)$ of the parent bronchus (local trunk, subscript $t$ ) and the sum of the cross-sectional areas $\left(\sum A_{b}\right)$ of the daughter bronchi (local branches, subscript $b$ ), is commonly equal to about 1.2 (Table 9). Although the airway caliber decreases with branching, the cumulated cross sectional area quickly increases.

Branches are currently unequal in size. The curved bronchi becomes shorter with a smaller bore with increased generation rank (Table 10). The number of generations leading to the pulmonary alveoli varies between the various territories.

The bronchioles arising from the terminal bronchi produce three to four generations down to the terminal bronchioles. The terminal bronchioles give birth to three successive generations of respiratory bronchioles, as described in the Weibel model. But, two to five gen- 
erations of respiratory bronchioles can exist. The last generation of respiratory bronchioles lead to alveolar ducts which conveys air to/from a set of alveolar sacs. Like the atrium of a roman house (domus) that leads to rooms, alveolar sacs are cavities bounded by a small variable number of alveoli.

The terminal and respiratory bronchioles belong to the pulmonary lobule, i.e., the region of the lung parenchyma ventilated by a bronchiole. A terminal bronchiole commonly divides into two branches, but one daughter duct can correspond to a terminal bronchiole, whereas the other is a respiratory bronchiole (anatomical and functional asymmetry).

The first respiratory bronchiole corresponds to the entrance of the pulmonary acinus, the basic structural compartment of gas exchange. The respiratory bronchioles are the first bronchioles along which alveoli appear for a minimal gas exchange. The nearer the alveolar duct, the higher the number of alveoli lining the bronchiolar wall. The wall-implanted alveoli are irregularly spaced.

The last in a series of respiratory bronchioles engenders the first generation of alveolar ducts. Alveolar ducts are completely covered by alveoli. The walls of alveolar ducts are, indeed, exclusively constituted by entry tips of alveoli. Five or more ramifications with wall alveoli close to each other can reside in the pulmonary acinus.

Terminal bronchioles correspond to the last bronchial generations that have a complete respiratory epithelium, i.e., last purely conducting airways. The number of terminal bronchioles is estimated to be about 28000 [80]. The pulmonary acinus is the elementary gas exchange compartment. The pulmonary alveolus is the gas exchange unit that appears on walls of acinal bronchioles, or respiratory bronchioles, distal to terminal bronchioles. The first generation of respiratory bronchioles marks the beginning of the respiratory compartment, from which gas exchange takes place. Incoming bronchioles at the acinus entrance divide into several generations. The last respiratory bronchiole generates a set of alveolar ducts, each endowed with 5 to 6 alveolar sacs.

The fractal geometry developed by Mandelbrot has been applied to the respiratory tract. Fractals are self-similar structures characterized by power-law functions and non-integer 
Table 11: Geometry of the six first generations of the Weibel model of the tracheobronchial tree (regular dichotomy) at $75 \%$ of the total lung capacity (Source: [81]).

\begin{tabular}{lllllll}
\hline Generation & $\begin{array}{l}L \\
(\mathrm{~mm})\end{array}$ & $\begin{array}{l}d_{h} \\
(\mathrm{~mm})\end{array}$ & $\begin{array}{l}R_{h} \\
(\mathrm{~mm})\end{array}$ & $L / d_{h}$ & $\begin{array}{l}A \\
\left(\mathrm{~mm}^{2}\right)\end{array}$ & $\begin{array}{l}\sum_{i} A \\
\left(\mathrm{~mm}^{2}\right)\end{array}$ \\
\hline 0 & 120 & 18 & 9 & 6.7 & 254 & 254 \\
1 & 47 & 12 & 6 & 3.9 & 113 & 226 \\
2 & 19 & 8 & 4 & 2.4 & 50 & 201 \\
3 & 7.5 & 5.6 & 2.8 & 1.4 & 25 & 197 \\
4 & 12 & 4.5 & 2.2 & 2.7 & 16 & 254 \\
5 & 10 & 3.5 & 1.7 & 2.9 & 10 & 308 \\
6 & 9 & 2.8 & 1.4 & 3.2 & 6 & 394 \\
\hline
\end{tabular}

dimensions (fractal dimension).

According to the Weibel model [81], in which the arborizing pattern assumes a regular dichotomy, twenty-two airway divisions exist from the trachea (generation zero [G0]) down to alveoli (Table 11). Down to G11, the lumen bore remains greater than $1 \mathrm{~mm}$ and the bronchus wall is composed of muscular and connective tissues with partial cartilage rings for the first generations, or complete ones for the following generations. Bronchi have cartilaginous walls, but not bronchioles. In adult lungs, the number of intrasegmental generations, walls of which contain cartilage varies between eight in the apical segment of the lower lobe and eleven in the lower lingular segment [82]. However, the number of branchings down to the last bronchial generation with a cartilaginous wall varies considerably.

\subsubsection{Heating and moistening}

The nose has an augmented exchange surface, which is highly vascularized, to operate as an air conditioner. It indeed warms and humidifies inhaled air. Inspired air can still be filtered, warmed, and humidified as it passes through the conducting airways before reaching the respiratory zone.

Therefore, the air properties within airways differ from that of ambient air. Air is heated at the body temperature $(T=310 \mathrm{~K})$ and saturated with water vapor. Consequently, the air density $(\rho)$ equals to $19.04 \times 10^{-6} \mathrm{~kg} \cdot \mathrm{m}^{-3}$, the dynamic viscosity $(\mu)$ to $1.068 \mathrm{~Pa} \cdot \mathrm{s}$, and the kinematic viscosity $(\nu)$ to $17.8 \times 10^{-6} \mathrm{~m}^{2} \cdot \mathrm{s}^{-1}$. 
Table 12: Compartments of an idealized 23-generation model of the tracheobronchial tree. Three zones are defined with their corresponding generations (Gi). Small intraparenchymal airways start with approximately generation G10. At the level of G10 (before G10 in short path form the trachea to alveolar ducts), the wall cartilage disappears and the number of smooth muscle cells increases.

\begin{tabular}{|c|c|c|}
\hline $\begin{array}{l}\text { Compartment } \\
\text { and content }\end{array}$ & $\begin{array}{l}\text { Involved } \\
\text { generations }\end{array}$ & Features \\
\hline$\overline{\text { (1) Conduction zone }}$ & G0-G16 & $\begin{array}{l}\text { Air convection } \\
\text { Anatomical dead space }\end{array}$ \\
\hline Trachea & G0 & Cartilaginous wall \\
\hline Main bronchi & \multicolumn{2}{|c|}{ Pulmonary lobe } \\
\hline Lobar bronchi & \multicolumn{2}{|c|}{ Pulmonary segment } \\
\hline Segmental bronchi & G3 & Cartilaginous wall \\
\hline Subsegmental bronchi & G4 & Cartilaginous wall \\
\hline Bronchi & G5-G8 & Cartilaginous wall \\
\hline Small bronchi & G9-G11 & \\
\hline Bronchioles & \multirow{2}{*}{\multicolumn{2}{|c|}{$\begin{array}{l}\text { Pulmonary lobule } \\
\text { G15-G16 }\end{array}$}} \\
\hline Terminal bronchioles & & \\
\hline \multicolumn{3}{|c|}{$\begin{array}{l}\text { Pulmonary acinus } \\
\text { Air transport by }\end{array}$} \\
\hline ion & G17-G19 & $\begin{array}{l}\text { Air transport by diffusion } \\
\text { Alveolized airway wall }\end{array}$ \\
\hline (3) Respiratory zone & $\begin{array}{l}\text { G20-G22, } \\
\text { alveoli }\end{array}$ & $\begin{array}{l}\text { Wall wholly dedicated to gas exchange } \\
\text { through the alveolocapillary membrane }\end{array}$ \\
\hline Respiratory bronchioles & G17-G19 & Moderate gas exchange \\
\hline $\begin{array}{l}\text { Alveolar ducts } \\
\text { Alveolar sacs }\end{array}$ & G20-G22 & Closed ends \\
\hline
\end{tabular}

\subsubsection{Airway function and air transport}

Functionally, the respiratory tract is composed of two main regions: the conducting airways, from upper airways to terminal bronchioles, and the respiratory zone with its respiratory bronchioles and alveolar ducts and sacs within pulmonary acini. The conducting zone is responsible for the anatomical dead space and the respiratory zone, for gas exchanges between air and blood (Table 12).

The volume of the conducting zone ( $\sim 150 \mathrm{ml}$ at rest, more during deep breathing) is much smaller than the volume of the respiratory zone $(\sim 2.5 \mathrm{l})$. The distance between terminal bronchioles and the end alveoli is short $(\sim 5 \mathrm{~mm})$ for efficient gas exchanges. 
Table 13: Values of the dimensionless flow governing parameters (Reynolds and Strouhal number using the mean $(\cdot)$ and peak $(\cdot)$ velocity and the bronchial radius and unsteady boundary layer $\left(\delta_{\mathrm{S}}\right)$, assuming a regular dichotomy and a sinusoidal time variation with a peak-to-peak breathing amplitude - the tidal volume $-V_{\mathrm{T}}=500 \mathrm{ml}$.

\begin{tabular}{lllllll}
\hline $\mathrm{G}$ & Sto & $\overline{R e}$ & $\widehat{R e}$ & $\widehat{R e}\left(\delta_{S}\right)$ & $\overline{S t}$ & $\widehat{S t}$ \\
\hline 0 & 2.7 & 389 & 612 & 232 & 0.018 & 0.011 \\
\hline 1 & 1.8 & 292 & 459 & 261 & 0.010 & 0.007 \\
\hline 2 & 1.2 & 219 & 344 & 293 & 0.006 & 0.004 \\
\hline 3 & 0.8 & 157 & 246 & 300 & 0.004 & 0.003 \\
\hline 4 & 0.7 & 97 & 153 & 232 & 0.005 & 0.003 \\
\hline 5 & 0.6 & 63 & 98 & 190 & 0.004 & 0.003 \\
\hline 6 & 0.4 & 39 & 61 & 150 & 0.004 & 0.003 \\
\hline
\end{tabular}

Consider rest breathing in an airway architecture characterized by a regular dichotomy. In the trachea $(\mathrm{G} 0)$, the inspiratory flow rate can be assumed to be sine wave $\left(q_{0}=\left(V_{\mathrm{T}} / 2\right) \omega \sin (\omega t)\right)$ with a peak-to-peak breathing amplitude - the tidal volume $\left(V_{\mathrm{T}}\right)$ - and angular frequency $\omega=2 \pi f(f=0.25 \mathrm{~Hz})$. The mean cross-sectional velocity $\left.\overline{V_{q}}=V_{\mathrm{T}} /(\pi A)\right)$. In branch $i$, the flow rate $\left(q_{i}\right)$ depends on the entry value $\left(q_{0}\right)$ according to $q_{i}=q_{0} / 2^{i}$. The values of the dimensionless flow governing parameter are listed for G0 to G6 in Table 13. During inspiration, unsteady convection in large intrathoracic airways is converted into a quasi-steady convection in small bronchi and, then, diffusion in pulmonary acini.

Air reaches alveolar sacs by gaseous diffusion. An ideal gaseous mixture, composed by $N$ gas species, is fully described by the mole fractions $\chi_{\ell}=n_{\ell} / n_{\text {tot }}\left(\sum_{\ell=1}^{N} n_{\ell}=n_{t o t}\right)$ of each species $\ell(\ell=1, \ldots, N)$ and the total concentration $c_{\text {tot }}$ of the mixture. The partial pressure of species $\ell$ in the mixture $\left(p_{\ell}\right)$ is given in the case of an ideal gas mixture by the equation of state $p_{\ell}=R_{\mathrm{g}} T c_{\mathrm{tot}} \chi_{\ell}\left(R_{\mathrm{g}}\right.$ : ideal gas constant; $T$ : absolute temperature). The force acting on species $\ell$ in a control volume is given by: $-\nabla p_{\ell}$. The force per mole of species $\ell$ that can be expressed as $-R_{\mathrm{g}} T \nabla \chi_{\ell} / \chi_{\ell}$ is balanced by the friction between species $\ell$ and $m$ acting on species $\ell: R_{\mathrm{g}} T \chi_{\ell}\left(v_{m}-v_{\ell}\right) / \mathcal{D}_{\ell m}\left(v_{\ell}\right.$ : molar average diffusion velocity of species $i$; $\mathcal{D}_{\ell m}$ : binary diffusion coefficient between the species $\ell$ and $m$ ). Each mole fraction that depends on time $(t)$ and space $(\mathbf{x})$ satisfies the continuity equation:

$$
\partial_{t} \chi_{\ell}+\nabla \cdot J_{\ell}=0,
$$


where $J_{\ell}=\chi_{\ell} v_{\ell}$ is the molar flux of species $\ell$. The Stefan-Maxwell diffusion is a model of gas diffusion in multicomponent mixtures [83]:

$$
-\nabla \chi_{\ell}=\sum_{m \neq \ell} \frac{\chi_{m} J_{\ell}-\chi_{\ell} J_{m}}{\mathcal{D}_{\ell m}} .
$$

At the alveolar wall, air and blood are separated by a thin barrier that has an extensive surface area. The structure of the alveolar air-blood barrier (thickness $0.62 \mu \mathrm{m}$ [84]) relies on:

(1) a pulmonary capillary endothelium with its basement membrane;

(2) a thin intertitium (thickness $100-140 \mathrm{~nm}) ;{ }^{35}$ and

(3) an alveolar epithelium with its basement membrane.

The air-blood barrier withstands changing hemodynamic pressures from the capillary side and surface tension forces from the alveolar side, as well as reacts to physical and chemical agents.

The conductance of the air-blood barrier for oxygen is correlated directly with its surface area and inversely with its thickness. The total resistance that oxygen molecules encounters is usually decomposed into resistance of the air-blood barrier, plasma, and erythrocyte. An average partial pressure difference of $57 \mathrm{~Pa}$ supports the transfer of oxygen across the air-blood barrier at a rate of $2.3 \times 10^{-5} \mathrm{~cm}^{2} / \mathrm{s}$ that is completed in $250-500 \mathrm{~ms}$ [84].

\subsubsection{Trans- and supercritical flow}

The respiratory tract contains two highly deformable materials: compressible air and distensible (and collapsible) airways. However, the air compressibility $\left(\mathrm{m}^{2} / \mathrm{N}\right)$ is much smaller (4 order of magnitude) than the bronchus distensibility $\left(\mathrm{m}^{2} / \mathrm{N}\right)$. Therefore, the contribution to the propagation speed of tiny pressure waves that results from air compressibility can

\footnotetext{
${ }^{35}$ In many parts of the air-blood barrier, the thickness of the lamina densa, a component of the basement membrane, located in the center of the intertitium is smaller than $50 \mathrm{~nm}[84]$.
} 
be neglected in simulations of fluid-structure interactions during forced breathing; the wave speed mainly relies only on airway distensibility. Its value is an order of magnitude less ( $c_{a w}$ : $\mathcal{O}[10 \mathrm{~m} / \mathrm{s}])$ than that computed with air compressibility $\left(\mathrm{c}_{\text {air }}: \mathcal{O}[100 \mathrm{~m} / \mathrm{s}]\right)$.

Forced expiration is an usual test carried out to quantify volumes and flow rates in humans. Starting form the total lung capacity, the subject exhales as fast as possible his entire vital capacity. In 50 to $150 \mathrm{~ms}$, a peak flow occurs, although the subject still yields energy to the respiratory apparatus. The observed flow limitation results from the appearance of a critical section $\left(M a_{\mathrm{aw}}=1\right)$ in the thoracic outlet segment of the trachea at the thoracic outlet $[41,86,86,87]$. Because the local cross-section area decays to a value of $20 \%$ of its initial value, the air flow velocity rises, whereas the wave speed decays abruptly. The flow can become supercritical $\left(M a_{\text {aw }}>1\right.$; the Mach number computed with the speed of pressure wave propagation in air rises to reach a relatively high value $\left.\left[M a_{\text {air }} \sim 0.5\right]\right)$.

\subsubsection{Airway surface fluid}

The apical surface of cells of the respiratory epithelium is covered by a triple-deck layer, i.e., the carbohydrate-rich glycocalyx coating and the double-layered airway surface liquid. The endoluminal liquid layer has two strata: a deep, aqueous, glycoprotein-free, lubricating region that bathes cilia - the epithelial lining fluid, also called the periciliary fluid or sol layer, and a superficial, viscous gel, or mucus layer (thickness 2-5 $\mu \mathrm{m}$ ). According to [88], the thickness of the airway surface liquid layer varies from about 3 to $7 \mu \mathrm{m}$. The epithelial lining fluid is more stable than the overlying mucus layer, which undergoes a relatively rapid turnover. Each layer is produced by a glandular apparatus: mucoid and serous secretions for the superficial and deep layer, respectively. The mucus and periciliary fluid layers are separated by a surfactant.

Surfactant regulates the surface tension and modify the mucus rheology. In addition, surfactant components enable sliding of mucus (gel phase) over the periciliary fluid (sol phase). Surfactant lowers adhesion at the gel-sol interface so that cilium tips can better penetrate the gel layer and more effectively propel this layer. Surfactant favors high ciliary 
beat frequencies, hence mucus speed.

Numerical investigations have been carried out on interactions between mucus motion and air flow at the microscopic scale [89]. Mucus displacement is driven by respiratory epithelium cilia, but not markedly on air stream. A quasi-steady Stokes flow of fluid with spatially variable viscosity has been simulated over a moving boundary [90]. A secondorder elliptic partial differential equation is non-linearly coupled to a first-order hyperbolic equation that describes the cilium beating, which takes into account the actin polymerization via a model of elastic beams of variable modulus.

\section{Mucociliary clearance}

The mucus layer entraps large deposited particles that are then transported by coordinated ciliary movements. Cilia freely beat in the periciliary fluid, but their tips contact the mucus layer during a part of the stroke.

Mucociliary clearance that relies on the continuous flow of airway surface liquid over surfaces of airway lumens prevents accumulation of inhaled contaminants and pathogens within the respiratory tract. Normal daily volume of respiratory secretion that arrives at the larynx is estimated to be about $10 \mathrm{ml}$, as its water content is partly reabsorbed due to ion transport. In healthy non-smoking adults, tracheal mucociliary clearance rate assessed by a radioisotope technique is 70 to $300 \mu \mathrm{m} / \mathrm{s}$ [91-93].

\section{Ciliary beat frequency and mucus speed}

Ex vivo, cilia of the nose, trachea, and large bronchi beat at a similar frequency $(10-20 \mathrm{~Hz})$. The ciliary beat frequency of samples of human respiratory epithelium ranges from 9.1 to $16.8 \mathrm{~Hz}[94]$. In patients examined by bronchoscopy, the ciliary beat frequency is similar in nasal, tracheal, and lower lobe bronchial epithelia $(14 \mathrm{~Hz})$, whereas it is slower in peripheral bronchi $(10 \mathrm{~Hz})$ [95]. Ciliary beat frequency in human nose in vivo is equal to $11.5 \pm 1.6 \mathrm{~Hz}[96]$ and that of maxillary sinus mucosa in vitro to $16.6 \pm 3.0 \mathrm{~Hz}$ [97]. Ciliary 
beat frequency hence slightly differs in vivo and in vitro.

The nasal mucus is displaced toward the pharynx at a speed of 1 to $3 \mathrm{~cm} / \mathrm{mn}$. Because the density of ciliated cells in the tracheobronchial tree decreases distally (from a proportion of about $22 \%$ in the trachea [G0] to $3 \%$ in G3), mucus speed ranges from $4.9 \mathrm{~mm} / \mathrm{mn}$ in the trachea to $1.7 \mathrm{~mm} / \mathrm{mn}$ in generation 3 .

\section{Mucus properties}

Surface interactions of the airway surface liquid with the respiratory epithelium on one side and airflow on the other rely on wettability (interfacial spreading capacity measured by the contact angle), adhesiveness (interfacial tension quantified by adhesivity) and tenacity (product of adhesive work and cohesivity). Cohesivity results from molecular attraction and depends on polymer length and density. Contact and rheological properties determine mucus transport function. The latter also depends on its permeability to particles. Mucus rheology deals with deformation and energy storage when a stress is applied and release of stored energy when the stress is removed. Restitution of stored energy allows mucus clearability.

At the macroscopic scale that yields averaged measures of rheological features, mucus has a time-dependent shear thinning (thixotropy) and viscoelastic behavior. Viscosity values of human respiratory mucus of 12 to $15 \mathrm{~Pa} \cdot \mathrm{s}$ with a relaxation time of about $40 \mathrm{~s}$ and an elastic modulus of 1 to $2 \mathrm{~Pa}$ is supposed to represent an optimal rheological profile for mucociliary clearance [98]. Mucus possess pseudoplasticity, elastothixotropy, spinability (capacity to be drawn out into long threads [Spinnbarkeit]) and adhesiveness [99].

Many factors contribute to mucus rheology, such as mucus glycoprotein types, hydration degree, and polymer structure [100]. The latter is influenced by $\mathrm{pH}$ and ion content in particular. The rheological properties of respiratory mucus vary according to the site along the airway network. Mucus heterogeneous microscopic domains contain entangled fibers and spaces filled by a low-viscosity fluid. For a given sampling site, mucus rheology depends not only on loading strength and application rate, but also on the degree of constituent crosslinking and entanglement. 


\section{Mucus permeability}

Airway surface liquid operates as a protective barrier at mucosal surfaces that traps and removes foreign particles such as bacteria and toxins (size $\sim 1 \mathrm{~mm}$ ) as well as viruses and environmental small particles (size 100-500 nm), but allows flux of nutrients, selected gases, ions, antibodies, and many proteins as well as cells of the mucosal immune system. The permeability threshold depends on the strength of association of mucin fibers that determines the typical mucin mesh size. Selective permeability of mucus is controlled by mucin environmental condition.

Mucus is characterized by heterogeneity of pore network of the viscoelastic matrix made of highly branched and strongly negatively charged mucus glycoproteins that are entangled with other mucosal constituents. Diffusion of amine-modified and carboxylated polystyrene particles (size 100-500 nm) that have neutral and negative surface charges at physiological $\mathrm{pH}$, respectively, has been investigated in sputum of mucoviscidosis patients by particle tracking with good spatial and temporal resolution (5 nm and $33 \mathrm{~ms}$ ) [101]. Small (size $<200 \mathrm{~nm}$ ), neutrally charged particles travel more quickly inside the mucus than large, charged particles. Large particles interact with a porous, charged, viscoelastic network. Assessed ensembleaverage diffusion coefficients of 100-, 200-, and 500-nm particles in sputum of cystic fibrotic patients range from about $1.5 \times 10^{-2}$ to $3.8 \times 10^{-4} \mu \mathrm{m}^{2} / \mathrm{s}$ [101] (in water, diffusion coefficients of 100-, 200-, and 500-nm particles are equal to 4.5, 2.3, and $0.9 \mu \mathrm{m}^{2} / \mathrm{s}$, respectively).

\section{Aerosol}

Aerosols are more or less concentrated suspension of tiny solid particles, liquid droplets, or their combinations in atmospheric air. In addition to natural aerosols, human activities generate about $10 \%$ of the total amount of aerosols in our atmosphere. Whereas inhaled pollutants and allergens can cause lung injuries and diseases, therapeutic aerosols pertain to the class of non- and minimally invasive treatments. This procedure targets either airway diseases or the systemic circulation to correct impaired functionning of cells remote from the ventilatory system. 
Inhalable particles are classified according to aerodynamic caliber: PM10 $(<10 \mu \mathrm{m})$, PM2.5 $(<2.5 \mu \mathrm{m})$, and nanoparticles $(<0.1 \mu \mathrm{m})$. Natural aerosols that range in size from $0.01 \mu \mathrm{m}$ to several tens of micrometers can mix. They are transported by atmospheric motions. Whereas particles of size $\mathcal{O}[1 \mu \mathrm{m}]$ deposit in upper airways, those of size $\mathcal{O}[1 \mathrm{~nm}]$ can reach alveoli.

Aerosol spray mainly corresponds to an aerosol mist of liquid particles delivered from a pressurized can with a valve and actuator. It contains a propellant liquid with boiling point slightly lower than room temperature in equilibrium with its vapor under pressure higher than atmospheric pressure. Two-component devices contain main and accessory chambers that store the main and a second component to deliver reactive mixture. Atomizers yield particles from pressurization by a hand-operated pump rather than a stored propellant.

Modeling of aerosol transport can rely on the coupling of Vlasov and Navier-Stokes equations [102]. The quantity of interest for aerosol is the probability density function $f\left(t, \mathbf{x}, \mathbf{v}_{i d}, r, \ldots\right)$. A first hypothesis states that the influence of the aerosol on the air flow is negligible. The quantities of interest for air stream are air density $(\rho)$, velocity $\mathbf{v}(t, \mathbf{x})$, and pressure $p(t, \mathbf{x})$; they are associated by the following set of equations:

$$
\left\{\begin{array}{l}
\partial_{t} \mathbf{v}+\nabla \cdot(\mathbf{v} \otimes \mathbf{v})+\frac{1}{\rho} \nabla p=\nu \nabla^{2} \mathbf{v}-\frac{1}{\rho} S \\
\nabla \cdot \mathbf{v}=0 \\
\partial_{t} f+\nabla \cdot\left(f \mathbf{v}_{i d}\right)+\nabla_{\mathbf{v}_{i d}} \cdot\left(f \mathbf{a}_{i d}\right)=0
\end{array}\right.
$$

where $S=\int_{\mathbf{v}_{i d}, r} m \mathbf{a}_{i d} f \mathrm{~d} \mathbf{v}_{i d} \mathrm{~d} r \quad$ and $\mathbf{a}_{i d}=D\left(\mathbf{v}-\mathbf{v}_{i d}\right)$ is the particle acceleration $(D$ : drag coefficient). ${ }^{36}$

\footnotetext{
${ }^{36}$ The drag coefficient $D$ is given by:

$$
D=\frac{3}{8} C_{d}\left(\operatorname{Re}_{\mathrm{drop}}\right) \frac{\rho_{\mathrm{air}}}{\rho_{\mathrm{drop}}} \frac{\left|\mathbf{v}-\mathbf{v}_{i d}\right|}{r} ; \quad \operatorname{Re}_{\mathrm{drop}}=\frac{2 r}{\mu} \rho_{\mathrm{drop}}\left|\mathbf{v}-\mathbf{v}_{i d}\right| .
$$
}




\subsection{Bioheat transfer in physiology and pathology}

The human body produces thermal energy that is exchanged with the ambiant air. The metabolic rate $\left(\mathrm{W} / \mathrm{m}^{2}\right)$ refers to the heat production by the human body. The basal metabolic rate is the minimal amount of energy released in an environment of thermal neutrality at rest, several hours after feeding. The total metabolic heat of a body can be calculated by multiplying the metabolic rate with the surface area of the human body. ${ }^{37}$

Three major mechanisms of heat exchange participate in the thermal energy balance convection, conduction, and radiation, in addition to a phase change, evaporation. The conservation of energy within the human body poises the internal production (sources) and loss (sinks; e.g., [105]):

$$
\Delta E=M-\left(W+Q_{\mathrm{conv}}+Q_{\mathrm{diff}}+Q_{\mathrm{rad}}+Q_{\text {evap }}+Q_{\mathrm{resp}}\right)
$$

where $\Delta E$ is the rate of energy storage in the body, $M$ the metabolic energy production, $W$ the external work, $Q_{\text {conv }}$ the surface heat loss by convection, $Q_{\text {diff }}$ that by diffusion, or heat conduction, $Q_{\text {rad }}$ that by radiation, and $Q_{\text {evap }}$ that by evaporation, and $Q_{\text {resp }}$ the respiratory heat loss.

The transfer of thermal energy ${ }^{38}$ by conduction between regions of a biological tissue results from a temperature gradient to reach a thermal equilibrium. The thermal energy results from random collisions between constituent particles of biomaterials.

The factors that can affect heat transfer in biological tissues can be classified into two categories. The first one includes heat sources from internal (cellular metabolism) and external origin (e.g., absorption of energy of incident waves), the role of which depends on the thermophysical properties of the target tissue. Most body heat is generated by the activity of the brain, liver, heart, and skeletal muscles. The second one comprises the generation

\footnotetext{
${ }^{37}$ The human body surface area (BSA) can be calculated using the formula of DuBois and DuBois [103, 104]: $B S A=0.007184 \mathrm{w}^{0.425} \cdot \mathrm{h}^{0.725}$

(w: body's weight $[\mathrm{kg}] ; \mathrm{h}$ : body's height $[\mathrm{cm}]$ ).

${ }^{38}$ The thermal energy of a material has the dimension of energy, but, unlike temperature, is not a state variable; its value does depend on the past history of the material.
} 
of heat fluxes due to conduction and forced convection (e.g., blood perfusion) of thermal energy.

\subsubsection{Thermoregulation}

Thermoregulation involves two opposite reflex — shivering and sweating — as well as change in the vasomotor tone - vasodilation and vasoconstriction. When the skin temperature is greater (lower) than that of the body's environment, the body can lose (gain) heat by radiation and conduction. The body can also eliminate heat by sweating (evaporative cooling). Conversely shivering, or shuddering, is set up in response to hypothermia; skeletal muscles shake to warm the body.

A large number of bioheat models has been developed to model the effect of blood flow on the heat transfer in living tissues [107]. A simplified thermoregulatory bioheat model was recently (2010) developed by coupling the Pennes equation and the Gagge's confort model [106].

\subsubsection{Therapeutic applications}

Heating at a temperature above $60^{\circ} \mathrm{C}$ severely damage proteins and cellular components and can cause cell death. As intuitively guessed, administration of a thermal dose is maximized by a high energy input during a short period [108].

Computational models of heat transfer during thermal ablation rely on the bioheat equation that includes an additional term associated with acoustic streaming. Further elevation of the temperature is required to produce an efficient heating in the presence of tissue perfusion in comparison to that in the absence of blood flow $[109,110]$.

Surgical resection of primary and metastatic tumors remains nowadays the golden standard of therapy, but cannot always be used. Therefore, alternative procedures have been proposed, such as artery infusion chemotherapy, percutaneous ethanol injection (PEI), cryother- 
apy, microwave coagulation therapy (MCT), and laser-induced thermotherapy (LITT). None of these therapies yieds long-term survival. Therapeutic procedures then focus on highfrequency radio-frequency (RF) and ultrasound (US) ablation. In particular, ultrasound enable atoms to move in unison to generate a mechanical wave, which can, in turn, be focused on small regions. During delivery of ultrasounds that can penetrate deep into the body's tissues, absorbed energy is transformed into thermal energy, hence the target tissue temperature rises, causing thermal coagulation and cell death.

In the past, temperature elevation in soft tissues was mostly modeled by the Pennes equation (Sect. 0.1.12). The heat source corresponds to the incident acoustic wave and heat sink to the blood flowing in capillaries. However, it looses its validity when the region of interest contains some large blood vessels. Cooling in large blood vessels and microvasculature need to be taken into account altogether. Incorporation of these two cooling sources increases the modeling complexity. In addition, the absorption of high-amplitude pressure waves in blood and vessel wall generates blood currents, the acoustic streaming.

\section{Tissue heating by electromagnetic waves}

Emission of electromagnetic waves destroys soft tissues, especially cancerous cells by acute heating associated with the absorption of the wave energy. Two major types of procedures comprise laser interstitial thermal therapy (LITT) and radiofrequency ablation (RFA). Laserbased therapy is usually performed in the near-infrared wavelength range $(700-2000 \mu \mathrm{m})$. When photons reach tissues, their energy can be converted into inter- and intramolecular energies. Another approach utilizes microwaves $(300 \mathrm{MHz}$ to $300 \mathrm{GHz})$, thus including the radio frequency spectrum. Polarized molecules generate a resulting dipole moment and can then rotate. Dipoles thus align with the alternating electric field of waves. The accompanied molecular friction between water and fat molecules can generate heat. However, this technique does not provoke an even heating of the target zone due to a heterogeneous distribution of the wave energy. During radiofrequency ablation, thermal energy is radiated from electrodes and propagates [111]. Heat radiation re-emitted from target organs is proportional to the square of the RF alternating current. The resistive heat from the ablation 
electrode thus decreases with the distance to the fourth power [112].

\section{Tissue heating by high-intensity focused ultrasounds}

Ultrasound with a frequency of several $\mathrm{MHz}$ and wavelength of a magnitude order of $1 \mathrm{~mm}$ can compress and expand tissues millions of times per second. Such a high-power ultrasound can penetrate deeply into tissues and be delivered to a small region without affecting significantly surroundings.

Because of the thermal loss due to viscous dissipation in tissues, finite-amplitude acoustic pressure can be computed in a soft, homogeneous medium using the non-linear Westervelt wave equation, when the medium is assumed to be a thermoviscous fluid [113]:

$$
\nabla^{2} p-\frac{1}{c^{2}} \partial_{t} p+\frac{\mathcal{D}_{\mathrm{p}}}{c^{4}} \partial_{t}^{3} p+\frac{K}{\rho_{0} c^{4}} \partial_{t}^{2} p^{2}=0
$$

where $\mathrm{c}$ is the characteristic medium sound speed, $\rho_{0}$ the medium density at rest, $\mathcal{D}_{\mathrm{p}}=2 c^{3} \alpha / \omega^{2}$ the acoustic diffusivity ( $\alpha$ : acoustic absorption coefficient; $\omega$ : angular frequency) and $K=$ $1+B / 2 A$ a non-linear coefficient, the term $B / 2 A$ reflecting the relation between the sound pressure and density.

Exposure of high-intensity focused ultrasounds affect the blood flow. Acoustic streaming $(\mathrm{S})$ is then added in the momentum conservation equation that governs blood flow [114]:

$$
D_{t} \mathbf{v}=-\frac{1}{\rho} \nabla p+\nu \nabla^{2} \mathbf{v}+\frac{1}{\rho} \mathrm{S}
$$

\section{References}

[1] Thiriet M, Graham JMR, Issa RI (1992) A pulsatile developing flow in a bend. Journal de Physique III 2:995-1013

[2] Handbook of Chemistry and Physics, Internet Version 2011, Lide DR (ed), (www.hbcpnetbase.com), 91st Edition, CRC Press/Taylor and Francis, Boca Raton

[3] Mendlowitz M (1948) The Specific Heat of Human Blood. Science 107:97-98 
[4] Woodcock JP (1976) Physical properties of blood and their influence on blood-flow measurement. Reports on Progress in Physics 39:65

[5] Jiang SC, Ma N, Li HJ, Zhang XX (2002) Effects of thermal properties and geometrical dimensions on skin burn injuries. Burns 28:713-717

[6] Nahirnyak VM, Yoon SW, Holland CK (2006) Acousto-mechanical and thermal properties of clotted blood. Journal of the Acoustical Society of America 119:3766-3772

[7] Holmes KR. Thermal conductivity of biomaterials (www.ece.utexas.edu/ valvano/research/Thermal.pdf)

[8] Womersley J (1955) Method for the calculation of velocity, rate of flow and viscous drag in arteries when the pressure gradient is known. The Journal of Physiology 127:553-563

[9] Quarteroni A, Ragni S, Veneziani A (2001) Coupling between lumped and distributed models for blood flow problems. Computing and Visualization in Science 4:111-124

[10] Smith NP, Pullan AJ, Hunter PJ (2002) An anatomically based model of transient coronary blood flow in the heart. SIAM Journal on Applied Mathematics 62:990-1018

[11] Olufsen MS, Peskin CS, Kim WY, Pedersen EM, Nadim A, Larsen J (2000) Numerical simulation and experimental validation of blood flow in arteries with structured-tree outflow conditions. Annals of Biomedical Engineering 28:1281-1299

[12] Laganà K, Dubini G, Migliavacca F, Pietrabissa R, Pennati G, Veneziani A, Quarteroni A (2002) Multiscale modelling as a tool to prescribe realistic boundary conditions for the study of surgical procedures. Biorheology 39:359-364

[13] Formaggia L, Gerbeau J-F, Nobile F, Quarteroni A (2002) Numerical treatment of defective boundary conditions for the Navier-Stokes equations. SIAM Journal on Numerical Analysis 40:376-401

[14] American Institute of Aeronautics and Astronautics (1998) Guide for the Verification and Validation of Computational Fluid Dynamics Simulations (G-077-1998e). AIAA Standards Series.

[15] ERCOFTAC Special Interest Group on Quality and Trust in Industrial CFD (2000). Best practice guidelines for industrial computational fluid dynamics. Lausanne, Switzerland: ERCOFTAC Coordination Centre

[16] Patankar SV (1980) Numerical Heat Transfer and Fluid Flow. Hemisphere Series on Computational Methods in Mechanics and Thermal Science, Hemisphere Publishing Corporation, Washington

[17] LeVeque RJ (2002) Finite Volume Methods for Hyperbolic Problems. Cambridge Texts in Applied Mathematics, Cambridge University Press, Cambridge, UK

[18] Pironneau O (1988) Méthodes des éléments finis pour les fluides. Masson, Paris and Finite element methods for fluids, John Wiley and Sons, Hoboken, NJ

[19] Girault V, Raviart PA (1986) Finite Element Methods for Navier-Stokes Equations: Theory and Algorithms. Springer, Berlin

[20] Brezzi F, Fortin M (1991) Mixed and Hybrid Finite Element Methods. Springer, New York

[21] Reddy JN (2006) An Introduction to the Finite Element Method. McGraw-Hill series in mechanical engineering, Engineering Series, McGraw-Hill Higher Education, New York

[22] Cebral JR, Löhner R (2001) From medical images to anatomically accurate finite element grids. International Journal for Numerical Methods in Engineering 51:985-1008

[23] Thiriet M, Brugières P, Bittoun J, Gaston A (2001) Computational flow models in cerebral congenital aneurisms I: Steady flow. Revue Mécanique et Industries 2:107-118

[24] Salmon S, Thiriet M, Gerbeau J-F (2003) Medical image-based computational model of pulsatile flow in saccular aneurisms. Mathematical Modelling and Numerical Analysis 37:663-679

[25] Milner JS, Moore JA, Rutt BK, Steinman DA (1998) Hemodynamics of human carotid artery bifurcations: computational studies with models reconstructed from magnetic resonance imaging of normal subjects. Journal of Vascular Surgery 1998:143-156 
[26] Moore JA, Rutt BK, Karlik SJ, Yin K, Ethier CR (1999) Computational blood flow modeling based on in vivo measurements. Annals of Biomedical Engineering 27:627-640

[27] Ladak HM, Milner JS, Steinman, DA (2000) Rapid 3D segmentation of the carotid bifurcation from serial MR images. Journal of Biomechanical Engineering 122:96-99

[28] Papaharilaou Y, Doorly DJ, Sherwin SJ, Peir J, Griffith C, Chesire N, Zervas V, Anderson J, Sanghera B, Watkins N, Caro CG (2002) Combined MRI and computational fluid dynamics detailed investigation of flow in idealised and realistic arterial bypass graft models. Biorheology 39:525-532

[29] Gill JD, Ladak HM, Steinman DA, Fenster A (2000) Accuracy and variability assessment of a semiautomatic technique for segmentation of the carotid arteries from 3D ultrasound images. Medical Physics 27:1333-1342

[30] Delfour MC, Zolesio JP (2011) Shapes and Geometries: Metrics, Analysis, Differential Calculus and Optimization, SIAM series on Advances in Design and Control, Society for Industrial and Applied Mathematics, Philadelphia, USA (second edition)

[31] Boissonnat J-D (1988) Shape reconstruction from planar cross-sections. Computer Vision, Graphics, and Image Processing 44:1-29

[32] Boissonnat J-D, Chaine R, Frey P, Malandain G, Salmon S, Saltel E, Thiriet M (2005) From arteriographies to computational flow in saccular aneurisms: the INRIA experience. Medical Image Analysis 9:133-143

[33] Boissonnat, J-D, Cazals F (2002) Smooth surface reconstruction via natural neighbour interpolation of distance functions. Computational Geometry 22:185-203

[34] Delingette H, Hébert M, Ikeuchi K (1992) Shape representation and image segmentation using deformable surfaces. Image and Vision Computing 10:132-144

[35] Taylor CA, Hughes TJR, Zarins CK (1998) Finite element modeling of blood flow in arteries. Computer Methods in Applied Mechanics and Engineering 158:155-196

[36] Peir J, Giordana S, Griffith C, Sherwin SJ (2002) High-order algorithms for vascular flow modelling. International Journal for Numerical Methods in Fluids 40:137-151

[37] Sherwin SJ, Peir J (2002) Mesh generation in curvilinear domains using high-order elements, International Journal for Numerical Methods in Engineering 53:207-223

[38] Giachetti A, Tuveri M, Zanetti G (2003) Reconstruction and web distribution of measurable arterial models. Medical Image Analysis 7:79-93

[39] Cohen LD (1991) On active contour models and balloons. Computer Vision, Graphics, and Image Processing 53:211-218

[40] Fetita C, Prêteux F, Beigelman-Aubry C, Grenier P (2004) Pulmonary airways: 3D reconstruction from multi-slice CT and clinical investigation, IEEE Transactions on Medical Imaging 23:1353-1364

[41] Thiriet M, Bonis M (1983) Experiments on flow limitation during forced expiration in a monoalveoalar model. Medical and Biological Engineering and Computing 21:681-687

[42] Thiriet M, Naili S, Ribreau C (2003) Entry length and wall shear stress in uniformly collapsed veins. Computer Modeling in Engineering \& Sciences 4:473-488

[43] Raghu R, Vignon-Clementel IE , Figueroa CA, Taylor CA (2011) Comparative study of viscoelastic arterial wall models in nonlinear one-dimensional finite element simulations of blood flow. ASME Journal of Biomechanical Engineering 133:081003

[44] Kim HJ, Vignon-Clementel I, Figueroa CA, Ladisa JL, Jansen KE, Feinstein J, Taylor CA (2009) On coupling a lumped parameter heart model and a three-dimensional finite element aorta model. Annals of Biomedical Engineering 37:2153-2169

[45] Causin P, Gerbeau JF, Nobile F (2005) Added-mass effect in the design of partitioned algorithms for fluid-structure problems. Computer Methods in Applied Mechanics and Engineering 194:42-44

[46] Fernández Varela MA, Gerbeau JF, Grandmont C (2007) A projection semi-implicit scheme for the coupling of an elastic structure with an incompressible fluid. International Journal for Numerical Methods in Engineering 69:794-821 
[47] Burman E, Fernández Varela MA (2009) Stabilization of explicit coupling in fluidstructure interaction involving fluid incompressibility. Computer Methods in Applied Mechanics and Engineering 198:766-784

[48] Boulakia M, Guerrero S (2010) Regular solutions of a problem coupling a compressible fluid and an elastic structure. Journal de Mathématiques Pures et Appliquées 94:341365

[49] Fernández Varela MA (2011) Coupling schemes for incompressible fluid-structure interaction: implicit, semi-implicit and explicit. SeMa Journal 55:59-108

[50] Wang K, Rallu A, Gerbeau JF, Farhat C (2011) Algorithms for interface treatment and load computation in embedded boundary methods for fluid and fluid-structure interaction problems. International Journal for Numerical Methods in Fluids 67:11751206

[51] Pennes HH (1948) Analysis of tissue and arterial blood temperatures in the resting human forearm. Journal of Applied Physiology 1:93-122

[52] Lighthill J (1978) Acoustic streaming. Journal of Sound and Vibration 61:391-418

53] Chien S (1970) Shear dependence of effective cell volume as a determinant of blood viscosity. Science 168:977-978

[54] Easthope PL, Brooks DE (1980) A comparison of rheological constitutive functions for whole human blood. Biorheology 17:235-247

[55] Chien S (1975), Biophysical behavior in suspensions. In: Surgenor D (ed) The red blood cell. Academic Press, New York

[56] Bucherer C, Lacombe C, Lelièvre J-C (1998) Viscosité du sang humain[Viscosity of the human blood]. In: Jaffrin MY, Goubel F (eds) Biomécanique des fluides et des tissus[Fluid and tissue biomechanics], Masson, Paris

[57] Joly M, Lacombe C, Quemada D (1981) Application of the transient flow rheology to the study of abnormal human bloods. Biorheology 18:445-452

[58] Thiriet M, Martin-Borret G, Hecht F (1996) Écoulement rhéofluidifiant dans un coude et une bifurcation plane symétrique. Application à l'écoulement sanguin dans la grande circulation[Shear-thinning flow in a bend and a symmetrical planar bifurcation. Application to the blood flow in large vessels]. Journal de Physique III 6:529-542

[59] Iolov A, Kane A, Bourgault Y, Owens R, Fortin A (2011) A finite element method for a microstructure-based model of blood. International Journal for Numerical Methods in Biomedical Engineering 27:1321-1349

[60] Paul R, Apel J, Klaus S, Schugner F, Shwindke P, Reul H (2003) Shear stress related blood damage in laminar couette flow. Artificial Organs 27:517-529

[61] Kunov MJ, Steinman DA, Ethier CR (1996) Particle volumetric residence time calculations in arterial geometries. Transactions of the ASME - Journal of Biomechanical Engineering 118:158-164

[62] Pinotti M, Rosa ES (1995) Computational prediction of hemolysis in a centrifugal ventricular assist device. Artificial Organs 19:267-273

[63] Designation: F 1841-97. Standard Practice for Assessment of Hemolysis in Continuous Flow Blood Pumps. Annual Book of ASTM Standards, Vol 13.01.

[64] Garon A, Farinas MI (2004) Fast three-dimensional numerical hemolysis approximation. Artificial Organs 28:1016-1025

[65] Giersiepen M, Wurzinger LJ, Opitz R, Reul H (1990) Estimation of shear stress-related blood damage in heart valve protheses - in vitro comparison of 25 aortic valves. International Journal of Artificial Organs 13:300-306

[66] Bludszuweit C (1995) Model for a general mechanical blood damage prediction. Artificial Organs 19:583-589

[67] Thiriet M (2008) Biology and Mechanics of Blood Flows, part II: Mechanics and Medical Aspects of Blood Flows. CRM Series in Mathematical Physics, Springer, New York 
[68] Segré G, Silberberg A (1962) Behaviour of macroscopic rigid spheres in Poiseuille flow I: Determination of local concentration by statistical analysis of particle passages through crossed light beam. Journal of Fluid Mechanics 14:115-135

[69] Segré G, Silberberg A (1962) Behavior of macroscopic rigid spheres in Poiseuille flow II: Experimental results and interpretation. Journal of Fluid Mechanics 14:136-157

[70] Leighton D, Acrivos A (1987) The shear-induced migration of particles in concentrated suspensions. Journal of Fluid Mechanics 181:415-439

[71] Koh CJ, Hookham P, Leal LG (1994) An experimental investigation of concentrated suspension flows in a rectangular channel. Journal of Fluid Mechanics 266:1-32

[72] Fahraeus R, Lindqvist T (1931) The viscosity of the blood in narrow capillary tubes. The American Journal of Physiology 96:562-568

[73] Michel CC, Curry FE (1999) Microvascular permeability. Physiological Reviews 79:703761

[74] Weinbaum S, Curry FE (1995) Modelling the structural pathways for transcapillary exchange. Symposia of the Society for Experimental Biology 49:323-345

[75] Agre P, Brown D, Nielsen S (1995) Aquaporin water channels: unanswered questions and unresolved controversies. Current Opinion in Cell Biology 7:472-483

[76] Tarbell JM, Demaio L, Zaw MM (1999) Effect of pressure on hydraulic conductivity of endothelial monolayers: role of endothelial cleft shear stress. Journal of Applied Physiology 87:261-268

[77] Chen SC, Liu KM, Wagner RC (1998) Three-dimensional analysis of vacuoles and surface invaginations of capillary endothelia in the eel rete mirabile. The Anatomical Record 252:546-553

[78] Thiriet M (2011) Cell and Tissue Organization in the Circulatory and Ventilatory Systems. Series "Biomathematical and Biomechanical Modeling of the Circulatory and Ventilatory Systems" (Vol. 1), Springer, Berlin

[79] Thiriet M (2011) Control of Cell Fate in the Circulatory and Ventilatory Systems. Series "Biomathematical and Biomechanical Modeling of the Circulatory and Ventilatory Systems" (Vol. 2), Springer, Berlin

[80] Wright JL, Thurlbeck WM (2005) Quantitative Anatomy of the Lung. In: Churg AM, Myers JL, Tazelaar HD, Wright JL (eds), Thurlbeck's Pathology of the Lung, third edition, Thieme, New York

[81] Weibel ER (1963) Morphometry of the human lung, Academic Press, New York

[82] Bucher U, Reid L (1961) Development of the intrasegmental bronchial tree: the pattern of branching and development of cartilage at various stages of intra-uterine life. Thorax $16: 207-218$

[83] Thiriet M, Douguet D, Bonnet JC, Canonne C, Hatzfeld C (1979) Influence du mélange $\mathrm{He}-\mathrm{O}_{2}$ sur la mixique dans les bronchopneumopathies obstructives chroniques. [Influence of a $\mathrm{He}-\mathrm{O}_{2}$ mixture on gas mixing in chronic obstructive lung diseases.] Bulletin Européen de Physiopathologie Respiratoire 15:1053-1068

[84] Maina JN, West JB (2005) Thin and strong! The bioengineering dilemma in the structural and functional design of the blood-gas barrier. Physiological Reviews 85:811-844

[85] Thiriet M, Bonis M, Adedjouma AS, Hatzfeld C, Yvon JP (1987) Experimental and theoretical models of flow during forced expiration: pressure and pressure history dependence of flow rate. Medical and Biological Engineering and Computing 25:551-559

[86] Thiriet M, Bonis M, Adedjouma AS, Yvon JP (1989) A numerical model of expired flow in a monoalveolar lung model subjected to pressure ramps. Journal of Biomechanical Engineering 111:9-16

[87] Thiriet M, Maarek JM, Chartrand DA, Delpuech C, Davis L, Hatzfeld C, Chang HK (1989) Transverse images of the human thoracic trachea during forced expiration. Journal of Applied Physiology 67:1032-1040

[88] Randell SH, Boucher RC (2006) Effective mucus clearance is essential for respiratory 
health. American Journal of Respiratory Cell and Molecular Biology 35:20-28

[89] Enault S., Lombardi D., Poncet P., Thiriet M. (2010) Mucus dynamics subject to air and wall motion, ESAIM: Proceedings 30:125-141

[90] Chatelin R, Poncet P, Didier A, Murris-Espin M, Anne-Archard D, Thiriet M (2012) Mucus and ciliated cells of the human lung: splitting strategies for particle methods and 3D Stokes flows. International Union of Theoretical and Applied Mechanics (IUTAM) Symposium on Particle Methods in Fluid Mechanics, October 15-17, 2012, Lyngby, Denmark

[91] Yeates DB, Pitt BR, Spektor DM, Karron GA, Albert RE (1981) Coordination of mucociliary transport in the human trachea and intrapulmonary airways. Journal of Applied Physiology: Respiratory, Environmental and Exercise Physiology 51:1057-1064

[92] Katz I, Zwas T, Baum GL, Aharonson E, Belfer B (1987) Ciliary beat frequency and mucociliary clearance. What is the relationship? Chest 92:491-493

[93] Wanner A (1981) Alteration of tracheal mucociliary transport in airway disease: effect of pharmacologic agents. Chest 80:867-870

[94] Yager J, Chen TM, Dulfano MJ (1978) Frequency of ciliary beats of human respiratory epithelium. Chest 73:627-633

[95] Rutland J, Griffin WM, Cole PJ (1982) Human ciliary beat frequency in epithelium from intrathoracic and extrathoracic airways. American Review of Respiratory Diseases 125:100-105

[96] Lindberg S, Runer T (1994) Method for in vivo measurement of mucociliary activity in the human nose. Annals of Otology, Rhinology and Laryngology 103:558-566

[97] Nuutinen J, Toskala ER, Saano V, Susanna J (1993) Ciliary beating frequency in chronic sinusitis. Archives of Otolaryngology - Head and Neck Surgery 119:645-647

[98] Lai SK, Wang Y-Y, Wirtz D, Hanes J (2009) Micro- and macrorheology of mucus. Advanced Drug Delivery Reviews 61:86-100

[99] Puchelle E, Zahm JM, Quemada D (1987) Rheological properties controlling mucociliary frequency and respiratory mucus transport. Biorheology 24:557-563

[100] King M (2006) Physiology of mucus clearance. Paediatric Respiratory Reviews 7:S212$\mathrm{S} 214$

[101] Dawson M, Wirtz D, Hanes J (2003) Enhanced viscoelasticity of human cystic fibrotic sputum correlates with increasing microheterogeneity in particle transport. Journal of Biological Chemistry 278: 50393-50401

[102] Boudin L, Desvillettes L, Motte R (2003) A modeling of compressible droplets in a fluid. Communications in Mathematical Sciences 1:657-669

[103] DuBois D, DuBois EF (1916) A formula to estimate the approximate surface area if height and weight be known. Archives of Internal Medicine 17:863-871

[104] Wang Y, Moss J, Thisted R (1992) Predictors of body surface area. Journal of Clinical Anesthesia 4:4-10

[105] Valvano JW (2006) Bioheat Transfer. In Webster JG (Ed.) Encyclopedia of Medical Devices and Instrumentation, 2nd edition, Wiley-Interscience, Hoboken, NJ

[106] Zolfaghari A, Maerefat M (2010) A new simplified thermoregulatory bioheat model for evaluating thermal response of the human body to transient environmrnt. Building and Environment 45:2069-2076

[107] Zolfaghari A, Maerefat M (2011) Bioheat transfer, Chap. 9: Developments in Heat Transfer. In dos Santos Bernardes MA (Ed.) Developments in Heat Transfer, Intech, Rijeka, Croatia

[108] Kuznetsov AV (2006) Optimization problems for bio-heat equation. International Communications in Heat and Mass Transfer 33:537-543

[109] Goldberg SN, Gazelle GS, Compton CC, McLoud TC (1995) Radiofrequency tissue ablation in the rabbit lung: efficacy and complications. Academic Radiology 48:776784 
[110] Gazelle GS, Goldberg SN, Solbiati L, Livoraghi T (2000) Tumor ablation with radiofrequency energy. Radiology 217:633-646

[111] Sheu TWH, Chou CW, Tsai SF, Liang PC (2005) Three-dimensional analysis for radiofrequency ablation of liver tumor with blood perfusion effect, Computer Methods in Biomechanics and Biomedical Engineering 8:229-240

[112] Lorentzen T (1996) A cooled needle electrode for radiofrequency tissue ablation: thermodynamic aspects of improved performance compared with conventional needle design. Academic Radiology 3:556-563

[113] Sheu TWH, Solovchuk MA, Chen AWJ, Thiriet M (2011) On an acoustics-thermalfluid coupling model for the prediction of temperature elevation in liver tumor. International Journal of Heat and Mass Transfer 54:4117-4126

[114] van der Zee J (2002) Heating the patient: a promising approach? Annals of Uncology $13: 1173-1184$ 